\title{
Remyelinating Oligodendrocyte Precursor Cell miRNAs from the Sfmbt 2 Cluster Promote Cell Cycle Arrest and Differentiation
}

\author{
Nicholas J. Kuypers, ${ }^{1,2 *} \oplus^{-}$Andrew N. Bankston, ${ }^{1,3 *}{ }^{\circledR}$ Russell M. Howard, ${ }^{1,3}$ Jason E. Beare, ${ }^{1,4}$ \\ and Scott R. Whittemore ${ }^{1,2,3}$ \\ ${ }^{1}$ Kentucky Spinal Cord Injury Research Center, Departments of ${ }^{2}$ Anatomical Sciences and Neurobiology and ${ }^{3}$ Neurological Surgery, and ${ }^{4}$ Cardiovascular \\ Innovation Institute, University of Louisville School of Medicine, Louisville, Kentucky 40292
}

Oligodendrocyte $(\mathrm{OL})$ loss contributes to the functional deficits underlying diseases with a demyelinating component. Remyelination by oligodendrocyte progenitor cells (OPCs) can restore these deficits. To understand the role that microRNAs (miRNAs) play in remyelination, $2^{\prime}, 3^{\prime}$-cyclic-nucleotide $3^{\prime}$-phosphodiesterase-EGFP ${ }^{+}$mice were treated with cuprizone, and OPCs were sorted from the corpus callosum. Microarray analysis revealed that Sfmbt2 family miRNAs decreased during cuprizone treatment. One particular Sfmbt2 miRNA, miR-297c-5p, increased during mouse OPC differentiation in vitro and during callosal development in vivo. When overexpressed in both mouse embryonic fibroblasts and rat OPCs (rOPCs), cell cycle analysis revealed that miR-297c-5p promoted $\mathrm{G}_{1} / \mathrm{G}_{0}$ arrest. Additionally, miR-297c-5p transduction increased the number of $01^{+}$rOPCs during differentiation. Luciferase reporter assays confirmed that miR-297c-5p targets cyclin T2 (CCNT2), the regulatory subunit of positive transcription elongation factor b, a complex that inhibits OL maturation. Furthermore, CCNT2-specific knockdown promoted rOPC differentiation while not affecting cell cycle status. Together, these data support a dual role for miR-297c-5p as both a negative regulator of OPC proliferation and a positive regulator of OL maturation via its interaction with CCNT2.

Key words: cuprizone; microarray; microRNA; oligodendrocyte progenitor cell; remyelination

\section{Significance Statement}

This work describes the role of oligodendrocyte progenitor cell (OPC) microRNAs (miRNAs) during remyelination and development in vivo and differentiation in vitro. This work highlights the importance of miRNAs to OPC biology and describes miR-297c$5 \mathrm{p}$, a novel regulator of OPC function. In addition, we identified CCNT2 as a functional target, thus providing a mechanism by which miR-297c-5p imparts its effects on differentiation. These data are important, given our lack of understanding of 0PC miRNA regulatory networks and their potential clinical value. Therefore, efforts to understand the role of miR-297c-5p in pathological conditions and its potential for facilitating repair may provide future therapeutic strategies to treat demyelination.

\section{Introduction}

Oligodendrocytes (OLs) are the only source of myelin in the CNS, and their loss is partially responsible for the dysfunctional

Received March 31, 2015; revised Dec. 18, 2015; accepted Dec. 22, 2015.

Author contributions: N.J.K., A.N.B., R.M.H., J.E.B., and S.R.W. designed research; N.J.K., A.N.B., R.M.H., and J.E.B. performed research; N.J.K., A.N.B., R.M.H., J.E.B., and S.R.W. analyzed data; N.J.K., A.N.B., and S.R.W. wrote the paper.

This research was supported by University of Louisville Grants RR15576/GM103507 and NS054708, The Commonwealth of Kentucky Challenge for Excellence, The Kentucky Spinal Cord and Head Injury Research Trust, and Norton Healthcare (S.R.W.). We thank Kariena Andres and Amberly Riegler for assistance with mouse colony maintenance; Dr. Vittorio Gallo for providing the CNPase-EGFP mice; Chris Worth, Aaron Puckett, and James McCracken for flow cytometry assistance; Allison Smith for cell culture assistance; Zachary Nicholas for assistance in staining quantification; Darlene Burke for biostatistical analyses; Drs. Michal Hetman and Sujata Saraswat Ohri for ongoing discussions of data and interpretations; Matthew Qiu for performing in situ hybridization experiments; and Dr. Pantelis Tsoulfas for critical comments on this manuscript.

The authors declare no competing financial interests. outcomes observed in individuals with multiple sclerosis (Miller et al., 2000), the leukodystrophies (Perlman and Mar, 2012), and spinal cord injury (Emery et al., 1998). Remyelination is driven by oligodendrocyte progenitor cells (OPCs), which remain mitotically competent within the mammalian CNS throughout adulthood with the ability to respond to OL death and myelin loss (Franklin and ffrench-Constant, 2008). However, following

\footnotetext{
*N.J.K. and A.N.B. contributed equally to this work.

Correspondence should be addressed to Dr. Scott R. Whittemore, Kentucky Spinal Cord Injury Research Center, Department of Neurological Surgery, 511 S. Floyd Street, MDR 616, University of Louisville School of Medicine, Louisville, KY 40292. E-mail: swhittemore@louisville.edu.

N.J. Kuypers' present address: Center for Translational Neuromedicine, University of Rochester Medical Center, Rochester, NY 14624.

DOI:10.1523/JNEUROSCI.1240-15.2016

Copyright $\odot 2016$ the authors $\quad 0270-6474 / 16 / 361698-13 \$ 15.00 / 0$
} 
extensive demyelination, remyelination fails (Waxman, 1989; Franklin and ffrench-Constant, 2008). The transcriptional events that regulate oligodendrocyte development are well understood as key regulatory elements such as Olig, Nkx, and Sox transcription factors have been identified (Wegner, 2008). However, posttranscriptional regulation by microRNAs (miRNAs) plays an equally important role in oligodendroglial maturation (Emery, 2010; Barca-Mayo and Lu, 2012; He et al., 2012). Elimination of miRNA processing in oligodendroglia via Dicer KO results in severe myelin deficits (Dugas et al., 2010). In addition, specific miRNAs have been identified both in vivo and in vitro to regulate OL differentiation (miR-138, miR-219, and miR-338; Dugas et al., 2010; Zhao et al., 2010; Birch et al., 2014) and OPC proliferation (miR-92 family, miR-124, and miR-9; Lau et al., 2008; Budde et al., 2010). However, the role of oligodendroglial miRNAs during remyelination in a cell-specific manner has not been described.

Here, we provide evidence supporting a role for OPC miRNAs during remyelination using the cuprizone model of demyelination and remyelination. Cuprizone is a copper chelator that induces CNS demyelination (most notably in the corpus callosum) when fed to mice (Mason et al., 2001; Matsushima and Morell, 2001; Steelman et al., 2012). Remyelination of the affected areas occurs upon removal of cuprizone from the diet (Mason et al., 2001; Matsushima and Morell, 2001). We hypothesized that changes in OPC miRNAs would accompany this response. Therefore, $2^{\prime}, 3^{\prime}$-cyclic nucleotide $3^{\prime}$-phosphodiesterase (CNPase)-enhanced green fluorescent protein-positive $\left(\mathrm{EGFP}^{+}\right) \mathrm{OPCs}$ were sorted from the white matter of cuprizone-treated and control transgenic mice, which display CNPase-EGFP specifically in oligodendroglia (Yuan et al., 2002), and miRNA expression levels were analyzed via microarray analysis. We identified multiple members of the Sfmbt2 miRNA family whose levels decrease during remyelination. One miRNA in particular, mmu-miR-297c-5p, was confirmed with quantitative real-time PCR (qRT-PCR) to increase concomitant with OPC differentiation in vitro and during postnatal development within the corpus callosum.

CCNT2 is a regulatory subunit of the multifunctional positive transcription elongation factor b (P-TEFb) complex (Peng et al., 1998). Heterodimerization of CCNT1/2 and Cdk9 (cyclindependent kinase 9), the catalytic subunit of P-TEFb, results in an activated form of the complex (Graña et al., 1994) with a broad range of biological activities, including cell cycle control, cytokine signaling, development, and cancer (De Luca et al., 1997; Simone et al., 2002; Bettencourt-Dias et al., 2004; Yang et al., 2008; Kohoutek, 2009). In OLs, P-TEFb has been implicated as a negative regulator of differentiation in zebrafish (Kim et al., 2012). Overexpression of miR-297c-5p antagonized cell cycle progression in mouse embryonic fibroblasts (MEFs) and rat OPCs (rOPCs) while promoting rOPC differentiation via repression of its target. Interestingly, despite the previously described roles for $\mathrm{P}-\mathrm{TEFb}$ in cell cycle, CCNT2-specific knockdown influenced only oligodendrocyte differentiation and not cell cycle. Collectively, our results identify miR-297c-5p as a regulator of oligodendroglia via repression of OPC cell cycle progression and introduce a novel miR-297c-5p-CCNT2 mechanism that enhances OL differentiation.

\section{Materials and Methods}

Animals. All animal procedures were performed in strict accordance with the Public Health Service Policy on Humane Care and Use of Laboratory Animals, Guide for the Care and Use of Laboratory Animals (Institute of Laboratory Animal Resources, National Research Council, 1996), and with the approval of the University of Louisville Institutional Animal Care and Use Committee and Institutional Biosafety Committee. $\mathrm{CNPase}_{\mathrm{EGFP}}{ }^{+}$mice were received from the laboratory of Dr. Vittorio Gallo (Children's National Medical Center, Washington, DC). These mice have the EGFP gene inserted downstream of both CNPase promoters C1 and C2 (Yuan et al., 2002), with expression enriched within oligodendroglia (Vogel et al., 1988). For genotyping, PCR of genomic DNA was performed using forward (5'-GGTGGTGCCCATCCTGGTCGA GC-3') and reverse (5'-CCAGCATGCCTGCTATTGTCTTCCC-3') primers (Life Technologies), and DNA was visualized with ethidium bromide. All CNPase-EGFP ${ }^{+}$pups were weaned at 3 weeks, and housed with CNPase-EGFP ${ }^{+}$littermates and other age-/sex-matched CNPase$\mathrm{EGFP}^{+}$litters for at least 5 weeks ( 8 weeks of age) before being included in the study.

Cuprizone treatment. Age- and weight-matched male and female $\mathrm{CNPase}-\mathrm{EGFP}^{+}$mice were fed ad libitum a diet of $0.2 \%(\mathrm{w} / \mathrm{w})$ oxalic bis-cyclohexylidenehydrazide (cuprizone; Sigma-Aldrich) mixed into normal chow (Harlan Teklad) beginning at 8 weeks of age. Equal gender distributions were maintained across all groups. Mice were distributed into six treatment groups as follows: groups 1 and 2, no cuprizone at 8 and 20 weeks of age; groups 3 and 4, 3 and 6 weeks of eating the cuprizone diet; and groups 5 and 6, 6 weeks of eating a cuprizone diet followed by 3 and 6 weeks of recovery while eating normal chow. During cuprizone treatment, animal weights were monitored weekly throughout the course of the experiment, and outliers were removed from the study. Any miRNAs identified as being significantly different between the 8- and 20 -week-old control groups were ruled out for further consideration as they may play a role in aging. For all other comparisons, the 8-week-old control group was used.

Tissue processing. Mice were killed with an overdose of $2.0 \%$ avertin (2,2,2-tribromoethanol) in sterile saline solution administered intraperitoneally before transcardial perfusion with cold PBS until the liver was clear and subsequent fixation with perfusion of cold $4 \%$ paraformaldehyde (PFA). Following fixation, brains were removed and postfixed for $24 \mathrm{~h}$ in $4 \%$ PFA. Cryoprotection was performed by immersion into a $30 \%$ sucrose solution for at least $72 \mathrm{~h}$ at $4^{\circ} \mathrm{C}$. Brains were blocked in tissuefreezing medium (Triangle Biomedical Sciences) and frozen at $-20^{\circ} \mathrm{C}$ before coronal sectioning with a Leica cryostat at $30 \mu \mathrm{m}$. Tissue sections were transferred to gelatin-coated slides and stored at $-20^{\circ} \mathrm{C}$.

$O P C$ isolation and differentiation assays. To assess the expression of miRNAs identified via array during in vitro differentiation, mouse OPCs (mOPCs) were isolated from the cortices of both male and female postnatal day 5 (P5) C57BL/6 mouse pups with magnetic activated cell sorting (Dincman et al., 2012). Briefly, brains were removed, and cortices were dissected away from whole brain and diced into $0.5 \mathrm{~mm}$ pieces by hand using a razor blade in $1 \mathrm{ml}$ of fresh HBSS. Tissue homogenates were collected into a $15 \mathrm{ml}$ conical tube and digested for $15 \mathrm{~min}$ at $37^{\circ} \mathrm{C}$ in enzyme mix 1 of the Neural Dissociation Kit (Miltenyi Biotec). Following mechanical dissociation with a wide-bore fire-polished Pasteur pipette, samples were digested with enzyme mix 2 of the Neural Dissociation Kit for $10 \mathrm{~min}$ at $37^{\circ} \mathrm{C}$ with gentle agitation. Next, tissue was further mechanically dissociated with a medium and small-bore fire-polished Pasteur pipette, successively, sieved through a $40 \mu \mathrm{m}$ cell strainer (BD Falcon, BD Biosciences), and then incubated at $37^{\circ} \mathrm{C}$ for an additional $10 \mathrm{~min}$ with agitation before centrifugation. The single cell number was counted with a hemocytometer, and the cell suspension was diluted 1:5 in cold HBSS with calcium and magnesium (Life Technologies), and centrifuged for 10 $\mathrm{min}$ at $300 \times g$. Next, the entire cell suspension was incubated for $7 \mathrm{~min}$ at $4^{\circ} \mathrm{C}$ with a mouse $\mathrm{O} 4$ hybridoma supernatant containing antibodies that recognize the $\mathrm{O} 4$ sulfatide. Following incubation, cells were washed and incubated with rat anti-mouse IgM magnetic beads (diluted $10 \%$ in Miltenyi buffer, Miltenyi Biotec), which ultimately bind only to $\mathrm{O}^{+}$ cells within the suspension. Following two washes, the magnetically bound cell are maintained in the column, while all $\mathrm{O}^{-}{ }^{-}$cells are eluted and discarded. $\mathrm{O}^{+}$cells were finally eluted and seeded at a density of $8000-12,000 \mathrm{mOPCs} / \mathrm{cm}^{2}$ into a pre-equilibrated $10 \mathrm{~cm}$ tissue culture dish coated with poly-D-lysine and laminin (both from Sigma-Aldrich) containing mOPC-A medium, which was prepared as follows: add 2.1 $\mathrm{g} / \mathrm{L} \mathrm{NaHCO}_{3}$ (Sigma-Aldrich) to DMEM-F12 lacking HEPES (Life Tech- 
nologies) and supplement with $1 \times \mathrm{N} 2,2 \times \mathrm{B} 27$, and $1 \%$ penicillin/streptomycin (all from Life Technologies); bovine serum albumin (BSA; $0.01 \%$, Sigma-Aldrich), $40 \mathrm{ng} / \mathrm{ml}$ fibroblast growth factor 2 (FGF2; Millipore), and $20 \mathrm{ng} / \mathrm{ml}$ PDGF $\alpha \alpha$ (Sigma-Aldrich). Average OPC yield was $1.5-3 \times 10^{5}$ cells/brain with $85-95 \%$ viability. Following isolation, cells were incubated at $37^{\circ} \mathrm{C}$ with $5 \% \mathrm{CO}_{2}$.

To assess the role of miRNAs identified via microarray analysis in differentiating primary mOPCs, $24 \mathrm{~h}$ postelution, and incubation in mOPC-A medium, cells were maintained in mOPC-A medium to drive proliferation or transferred to mOPC differentiation medium for $72 \mathrm{~h}$. mOPC differentiation medium was prepared as follows: add $2.1 \mathrm{~g} / \mathrm{L}$ $\mathrm{NaHCO}_{3}$ (Sigma-Aldrich) to DMEM-F12 lacking HEPES (Life Technologies) and supplement with $1 \times \mathrm{N} 2,2 \times \mathrm{B} 27,1 \%$ penicillin/streptomycin (all from Life Technologies), BSA (0.01\%), and T3 (40 ng/ml; both from Sigma-Aldrich). Following experimental condition assignment and the $72 \mathrm{~h}$ differentiation or proliferation period, total RNA was collected from each plate. Total RNA was also collected immediately following the $24 \mathrm{~h}$ postelution time point from a subset of plates to serve as a calibration control for qRT-PCR experiments. A single plate is considered $n=1$, and each condition (72 h mOPC-A medium, $72 \mathrm{~h}$ mOPC differentiation medium, or the $24 \mathrm{~h}$ mOPC-A medium calibration sample) was replicated to achieve $n=4$ for qRT-PCR-based experiments and $n=3$ for immunocytochemistry (ICC) assessment of oligodendroglial differentiation markers.

To assess the effect of miR-297c overexpression during differentiation, $\mathrm{O}^{+}{ }^{+}$rOPCs were immunopanned from the spinal cord of adult Fischer 344 rats that ubiquitously express human placental alkaline phosphatase, as detailed previously (Cao et al., 2010). Briefly, the dissected spinal cords were minced into $1 \mathrm{~mm}^{3}$ pieces, and incubated in HBSS containing $0.1 \%$ papain, $0.1 \%$ neutral protease, and $0.01 \% \mathrm{DNase}$ for $30 \mathrm{~min}$ at $37^{\circ} \mathrm{C}$. The digestion was stopped by the addition of an equal volume of DMEM containing 20\% fetal bovine serum (FBS). Tissues were dissociated by repeated trituration with fire-polished Pasteur pipettes and were filtered through $70 \mu \mathrm{m}$ nylon mesh. The cells were incubated on an anti-RAN-2 antibody-coated dish for $30 \mathrm{~min}$ to deplete type 1 astrocytes and meningeal cells, and then were transferred to an O4-coated dish for $45 \mathrm{~min}$ to select OPCs. The purified OPCs on the dish were removed with trypsin and cultured in poly-L-lysine/laminin-coated dishes with DMEM/F12 medium containing $1 \times \mathrm{N} 2$ and $1 \times \mathrm{B} 27$ supplements, FGF2 $(20 \mathrm{ng} / \mathrm{ml})$, PDGF $\alpha \alpha(10 \mathrm{ng} / \mathrm{ml})$, insulin $(5 \mu \mathrm{g} / \mathrm{ml})$, and BSA $(0.1 \%)$. Cells were fed with fresh growth medium every other day. In all cases, an aliquot of cells was analyzed the next day to determine the efficiency of the immunopanning. Only those cell preparations in which $>95 \%$ of the bound cells expressed $\mathrm{O} 4$ were used in the experiments. For differentiation assays, rOPC cultures were transduced with control (catalog \#S02-005000-01, Dharmacon) or miR-297c (catalog \#VSM6213-213640643, Dharmacon) lentivirus at a multiplicity of infection of 5 or with control (catalog \#SHC016V, Sigma-Aldrich) or CCNT2 shRNA (catalog \#SHCLNVNM_028399, Sigma-Aldrich) overnight. rOPC culture medium was changed to differentiation medium (rOPC culture medium without growth factors, supplemented with T3) $24 \mathrm{~h}$ after transduction. Cultures were differentiated for $3 \mathrm{~d}$ before staining.

Immunohistochemistry and immunocytochemistry. To distinguish $\mathrm{CNPase}_{\mathrm{EGFP}}{ }^{+}$-expressing cells from neurons and other glia, sections were stained with primary antibodies against the intermediate filaments glial fibrillary acidic protein (GFAP) and neurofilament medium subunit (NF-M; $145 \mathrm{kDa}$ ) to identify astrocytes and neurons, respectively, and EGFP to identify oligodendroglial lineage cells. Staining protocols were as follows: tissue sections were blocked with 5\% BSA, 10\% normal donkey serum (NDS), and $0.1 \%$ Triton X-100 in Tris-buffered saline (TBS) for $1 \mathrm{~h}$ at room temperature. Primary polyclonal antibodies NF-M (rabbit, 1:250; Millipore), GFAP (rabbit, 1:500; Dako), EGFP (chicken, 1:100; Abcam), and NG2 (rabbit, 1:50; Millipore Bioscience Research Reagents) were incubated for $24 \mathrm{~h}$ at $4^{\circ} \mathrm{C}$ with $5 \% \mathrm{BSA}, 5 \% \mathrm{NDS}$, and $0.1 \%$ Triton $\mathrm{X}-100$ in TBS. Sections were next washed at room temperature $(3 \times)$ with TBS and incubated with fluorescein isothiocyanate (FITC; 1:200)conjugated or rhodamine [tetramethylrhodamine isothiocyanate (TRITC); 1:200]-conjugated $\mathrm{F}\left(\mathrm{ab}^{\prime}\right) 2$ secondary antibodies (donkey; Jackson ImmunoResearch) for $1 \mathrm{~h}$ at room temperature. Species-specific IgG iso- type controls were used to account for any nonspecific binding or other cellular protein interactions. Hoechst staining was used to identify nuclei. Fluorescent imaging was performed on a Nikon Eclipse TE 300 inverted microscope with a spot CCD camera. Exposure times remained identical across all images. Callosal regions of interest (ROIs) were selected in serial cross sections at $-2.0 \mathrm{~mm}$ from bregma by tracing the corpus callosum from midline to the lateral extent of the corpus callosum. Bilateral EGFP intensity within the selected ROI of CNPase-EGFP ${ }^{+}$ brains was used to estimate the relative decrease and return in CNPaseEGFP expressing oligodendroglia during cuprizone demyelination and remyelination, respectively. ROI selection and EGFP intensity quantification was performed with Elements software (Nikon), and one-way ANOVA with Tukey's post-test was performed ( $n=3 /$ cuprizone treatment condition). Callosal CNPase-EGFP and NG2 coexpression was captured at $60 \times$ by confocal microscopy using an Olympus FV1000 Confocal Microscope and FluoView software. Orthogonal slices were generated using the Nikon NIS-Elements software.

For mouse and rat OPC differentiation experiments, staining against the surface markers A2B5, O4, and O1 was used to assess the oligodendroglial ontogenetic stage, and GFAP was used to identify astrocytes. The staining protocol was as follows: cells were washed with the appropriate culture medium and incubated with hybridoma supernatant (A2B5, O4, or O1) diluted to $70 \%(\mathrm{v} / \mathrm{v})$ in $20 \%$ culture medium, and $10 \%$ NDS for 45 $\min$ at $4^{\circ} \mathrm{C}$. Following washes $(3 \times)$ with culture medium, cells were fixed with $4 \%$ PFA at room temperature for $10 \mathrm{~min}$ and washed $(3 \times)$ with Dulbecco's PBS (DPBS) before overnight incubation with GFAP (rabbit, 1:500; Dako) at $4^{\circ} \mathrm{C}$ diluted in blocking solution containing $10 \%$ NDS, $0.5 \%$ BSA, and $0.1 \%$ Triton X-100 in DPBS. The following morning, cells were washed $(3 \times)$ with DPBS and incubated with FITC-conjugated (1: $200)$ or TRITC-conjugated (1:200) F( $\left.\mathrm{ab}^{\prime}\right) 2$ secondary antibodies (donkey; Jackson ImmunoResearch) for $1 \mathrm{~h}$ at room temperature. Speciesspecific IgG isotype controls were used to account for any nonspecific binding or other cellular protein interactions. Hoechst staining was used to identify nuclei. Photomicrographs were captured using a Nikon TiE 300 Inverted Microscope with a DXM-1200C coded digital camera and NIS-Elements Software (Nikon). Images were randomized and counts were performed by a laboratory member blinded to group. Percentages of $\mathrm{A} 2 \mathrm{~B}^{+}{ }^{+} / \mathrm{Hoechst}^{+}, \mathrm{O}^{+}{ }^{+} / \mathrm{Hoechst}^{+}, \mathrm{O}^{+} /$Hoechst $^{+}$, or $\mathrm{GFAP}^{+} /$ Hoechst $^{+}$cells per total Hoechst ${ }^{+}$nuclei were used for quantification. One-way ANOVA with Bonferroni post-test was performed for miR297c overexpression, and two-way ANOVA with Tukey's post-test was performed for CCNT2 knockdown. Data are presented as a mean percentage $\pm \mathrm{SD}(n=3)$.

Callosal dissection and tissue dissociation. For microarray experiments, the corpora callosa of $10 \mathrm{CNPase}_{\mathrm{EGFP}}{ }^{+}$mice within the same experimental group were pooled to yield a single sample and were collected in replicates of $n=3$. A sample from two wild-type (WT) mice that were subject to the same dissection and digestion protocol served as a negative control to EGFP expression in fluorescence-activated cells sorting (FACS) experiments. Mice were anesthetized with $2.0 \%$ avertin in sterile saline solution administered intraperitoneally before transcardial perfusion with cold PBS. Following perfusion, brains were removed and the corpus callosum from bregma to $-2.5 \mathrm{~mm}$ from bregma was dissected away from surrounding cortical and striatal tissue in cold HBSS lacking calcium and magnesium (Life Technologies). Following dissection, corpora callosa were diced by hand using a razor blade in $1 \mathrm{ml}$ of fresh HBSS. Tissue homogenates were collected into a $15 \mathrm{ml}$ conical tube and digested for $15 \mathrm{~min}$ at $37^{\circ} \mathrm{C}$ in enzyme mix 1 of the Neural Dissociation Kit (Miltenyi Biotec). Following mechanical dissociation with a wide-bore fire-polished Pasteur pipette, samples were digested with enzyme mix 2 of the Neural Dissociation Kit for $10 \mathrm{~min}$ at $37^{\circ} \mathrm{C}$ with gentle agitation. Next, tissue was further mechanically dissociated with a medium-bore and small-bore fire-polished Pasteur pipette, successively, sieved through a $40 \mu \mathrm{m}$ cell strainer (BD Falcon, BD Biosciences), and then incubated at $37^{\circ} \mathrm{C}$ for an additional $10 \mathrm{~min}$ with agitation before centrifugation. The single-cell number was counted with a hemocytometer. The cell suspension was diluted 1:5 in cold HBSS with calcium and magnesium (Life Technologies) and centrifuged for $10 \mathrm{~min}$ at $300 \times \mathrm{g}$. Cell pellets were resuspended in $8.5 \mathrm{ml}$ of buffer stock (Miltenyi Biotec) con- 
Table 1. Sfmbt2 family miRNAs identified in remyelinating OPCs

\begin{tabular}{|c|c|c|c|c|}
\hline miRNA ID & Mature sequence & $\begin{array}{l}\text { Repeats in } \\
\text { cluster }\end{array}$ & $\begin{array}{l}\text { Log2 } \mathrm{FC} \text { value } \\
\text { ( } 6 \text { weeks vs control) }\end{array}$ & $\begin{array}{l}p \text { value (Benjamini- } \\
\text { Hochberg correction) }\end{array}$ \\
\hline mmu-miR-297a-5p & AUGUAUGUGUGCAUGUGCAUGU & 5 & -1.50 & 4.54E-05 \\
\hline mmu-miR-297b-5p & AUGUAUGUGUGCAUGA ACAUGU & 1 & +1.35 & $5.79 \mathrm{E}-02$ \\
\hline mmu-miR-466b-5p & UGAUGUGUGUGUACAUGUACAU & 7 & -2.28 & $2.45 \mathrm{E}-05$ \\
\hline mmu-miR-467a-5p & UACAGUGCC $\bar{C} \cup \overline{G C A U G U \cup A U G C G}$ & 9 & NA & NA \\
\hline mmu-miR-669a-5p & AGUUGUGUGUGGAUGUC $\overline{A U G U C U}$ & 12 & -1.04 & $5.64 \mathrm{E}-03$ \\
\hline mmu-miR-466b-3p & AUACAUACA CGCACACAUAAGA & 7 & -2.09 & $3.41 \mathrm{E}-05$ \\
\hline mmu-miR-467a-3p & CAUAUACAUACACACAC CUACA & 9 & -2.37 & $1.82 \mathrm{E}-05$ \\
\hline mmu-miR-669a-3p & ACAUAACAUACA CACACACGUAU & 12 & -2.45 & $2.45 \mathrm{E}-05$ \\
\hline
\end{tabular}

The mature sequences, log 2 fold change (FC) values, and FDR $p$ values are shown for the $S f m b t 2$ family miRNAs identified in remyelinating OPCs that are most highly represented on the genome. Sequence similarity across $3 p$ and $5 p$ miRNA sequences is high, and common bases are underlined.

taining $0.5 \%$ BSA and $5 \mu \mathrm{g} / \mathrm{ml}$ insulin, which was added to $5.7 \mathrm{ml}$ of $90 \%$ Percoll (Sigma-Aldrich) in $10 \times$ HBSS and centrifuged at $15,000 \times g$ for $15 \mathrm{~min}$ to remove myelin. Following centrifugation, myelin was aspirated from the top, and the middle glial layer of cells was removed and centrifuged at $1200 \times g$ for $10 \mathrm{~min}$ to remove the Percoll. Cells were washed twice and resuspended in a final volume of $0.5 \mathrm{ml}$ of buffer stock (Miltenyi Biotec) containing BSA and insulin in preparation for FACS. For white matter development studies, C57BL/6 pups at 1,2, and 3 weeks of age were perfused, and corpora callosa were dissected, as stated above, in replicates of $n=3 /$ time point.

FACS. FACS was performed using a MoFlo system (DAKO). Cells remained on ice throughout all sorting experiments. The triggering setting was reconfigured for cell size and fluorescence for each experiment. Live analysis of the pulse width versus forward scatter excluded doublets. When sorting CNPase-EGFP ${ }^{+}$cells, event triggering was based on the fluorescence of endogenous EGFP at an excitation of $488 \mathrm{~nm}$ and detection at 530/40 nm. To assess the presence of any myeloid lineage cells in sorted populations, triggering was based on common leukocyte antigen CD45-conjugated peridinin chlorophyll protein (perCP) fluorescence (1:100; BD Biosciences) at an excitation of $488 \mathrm{~nm}$ and detection at 678 $\mathrm{nm}$. To establish background expression and gating parameters, callosal tissue from WT mice was used as a negative control for EGFP expression, and perCP-conjugated IgG isotype control (1:100; BD Biosciences) was used for CD45-perCP expression. Dissociated cell suspensions were incubated with primary antibodies for $30 \mathrm{~min}$ on ice in buffer (Miltenyi Biotec) with $0.5 \%$ BSA and $5 \mu \mathrm{g} / \mathrm{ml}$ insulin before washing $1 \times$ with cold DPBS. Cells were resuspended in cold buffer (Miltenyi Biotec) lacking serum for sorting.

Microarray analysis. Sorted cells were centrifuged at $300 \times g$ for 10 $\mathrm{min}$, and the supernatant was aspirated. Total RNA was extracted from cell pellets immediately following sorting using the mirVana miRNA Isolation Kit (Life Technologies) as per the manufacturer protocol. Eluted total RNA was immediately frozen and stored at $-80^{\circ} \mathrm{C}$. Once all microarray samples were collected ( $n=3$ /group), samples were shipped on dry ice to Exiqon where a single-color (Hy5), genome-wide miRNA expression profile analysis was performed using miRCURY LNA miRNA Arrays. Exiqon performed all miRNA quality control, extraction, enrichment, and normalization, and the data were received as expression values above background. Differentially expressed miRNAs were identified statistically by group ANOVAs performed across cuprizone treatment conditions with Benjamini-Hochberg correction for a $10 \%$ false discovery rate (FDR; Reiner et al., 2003). Three-dimensional principal component analysis (PCA) and unsupervised hierarchical clustering across both sample and miRNA expression values were performed using log2transformed fold change values of the 97 most differentially expressed miRNAs versus controls (top 40\%). All analyses were performed with the software package R (public domain; http://www.r-project.org). To establish a functional role for Sfmbt2 miRNA downregulation at 6 weeks of cuprizone treatment, the miRanda algorithm (www.microrna.org) was used to generate a list of targets common to each of the miRNAs listed in Table 1. Gene ontology (GO) analysis was performed using ingenuity pathway analysis on target genes with a mirSVR score cutoff of -1.3 (lower values represent more reliability in target prediction). For identifying targets of miR-297c-5p specifically, we used miRanda, miRDB (www.mirdb.org), and Target Scan (www.targetscan.org) to identify predicted target-seed sequence interactions common to each algorithm. Conservation of the miR-297c-5p-CCNT2 interaction in humans was assessed with miRanda.

miR-297c-CCNT2 targeting validation. To generate the Luciferase reporter containing the CCNT2 3'UTR, the full-length CCNT2 3'UTR was amplified from mouse genomic DNA by nested PCR using the following primers: 5' -CTCGCTGCTAAGTGCCCAAGG-3' (outer forward); 5' GCCAGTTCCAAGTGAATTCGAGG-3' (outer reverse); 5'-GTGTGT CTCGAGTAATGAAAGCTTCCTTTGTTTAGGTCAACTT-3' (inner forward); and 5'-GTGTGTCTCGAGTCTGTGGTTATTATTGTCAGG-3' (inner reverse). The PCR product was digested by XhoI and ligated downstream of the firefly luciferase coding sequence in pcDNA (provided by Yue Feng, Emory University, Atlanta, GA). Mutagenesis of the sequence in the CCNT2 $3^{\prime}$ UTR complimentary to the miR-297c seed region was performed using the Quick Change Lightning Mutagenesis Kit (Agilent) and the following primers: 5'-GTATTTATGGTAATAT CTAAACATACAAAAATCTTTCTACATTTTTCTCCACTTTCTTGTA AACAGCTG-3' (forward); and 5'-CAGCTGTTTACAAGAAAGTGGA GAAAAATGTAGAAAGATTTTTGTATGTTTAGATATTACCATAAA TAC-3' (reverse). HEK 293FT cells were reverse transfected with $100 \mathrm{ng}$ of firefly luciferase (empty vector, CCNT2 3'UTR, or mutant CCNT2 3'UTR), 20 ng of renilla luciferase (provided by Yue Feng, Emory University), and $75 \mathrm{~nm}$ control (catalog \#4464059, Life Technologies) or mmu-miR-297c mimic (catalog \#4464066, Assay MC12307, Life Technologies) using RNAiMax (Life Technologies). Luciferase readings were measured $24 \mathrm{~h}$ after transfection using the Dual Luciferase Reporter Assay (Promega), and levels in miR-297c-5p mimic transfected cells were quantified as a percentage of the levels in control mimic transfected cells. Data were analyzed by one-way ANOVA with Bonferroni post-test and are presented as the mean percentage $\pm \mathrm{SD}(n=3)$.

$q R T-P C R$. All qRT-PCR experiments were performed using an ABI 7900HT real-time PCR instrument (Life Technologies). For mOPC differentiation and white matter development experiments, total RNA was extracted with the mirVana miRNA Isolation Kit (Life Technologies) as per the manufacturer protocol. RNA concentrations were estimated with a NanoDrop 5000 machine with RNA elution buffer as a blank. For phenotypic mRNA analysis, first-strand synthesis of cDNA was performed using the RT ${ }^{2}$ First Strand Kit, and $\mathrm{RT}^{2}$ SYBR Green ROX Master Mix (both from Qiagen) was used for qRT-PCR. Mouse-specific mRNA primer assays are as follows: oligodendrocyte transcription factor 2 (Olig2; PPM04752B), platelet-derived growth factor receptor $\alpha$ (PDGFR $\alpha$; PPM03640D), proteolipid protein 1 (PLP1; PPM04717C), and glyceraldehyde-3-phosphate dehydrogenase (Gapdh; PPM02946E; all purchased as predesigned primer sets from Qiagen), while custom Invitrogen primers were used to access gene expression levels for myelin basic protein (MBP; forward primer $5^{\prime}$-GCCCATTGGTACACACT AAC- $3^{\prime}$ and reverse primer $5^{\prime}$-CTCGATTCAGTGACAGGAAC- $3^{\prime}$ ), 
GFAP (forward primer $5^{\prime}$-TCCTGGAACAGCAAAACAAG-3' ${ }^{\prime}$, and reverse primer $5^{\prime}$-CAGCCTCAGGTTGGTTTCAT-3'), microtubuleassociated protein 2 (Map 2; forward primer 5'-GAACTGACTCT CTCTGGATCTG-3' ${ }^{\prime}$, and reverse primer $5^{\prime}$-ACCTTCAGCCAAACT GAGC-3'). Data were analyzed as a percentage of the control corpus callosum by one-way ANOVA with Tukey's post-test and are presented as the mean $\pm \mathrm{SD}(n=3)$. For mOPC differentiation and callosal development experiments, miRNA reverse transcription (RT) was performed with the TaqMan MicroRNA Reverse Transcription Kit (Life Technologies) in combination with $5 \times$ miRNA-specific RT primers, and qRTPCR analysis was performed by adding miRNA-RT product to TaqMan Universal PCR Master Mix (Life Technologies). TaqMan 20× miRNA primer assays were as follows: mmu-miR-338-5p, mmu-miR-297c-5p, U6 small nucleolar RNA (U6 snRNA), and small nucleolar RNA 135 (snoRNA 135; all purchased from Life Technologies). For all qRTPCR experiments, target and reference gene amplification was performed in separate tubes and run in triplicate. RNA levels were quantified using the $\Delta \Delta \mathrm{CT}$ method. For normalization, Gapdh was used for SYBR Green ROX-based experiments, and U6 snRNA or snoRNA135 was used for TaqMan-based experiments. Data were analyzed by one-way ANOVA with Tukey's post-test and are presented as the mean $\pm \mathrm{SD}(n=4)$.

Cell cycle analyses. To assess the role of miR-297c-5p mimic transfection on cell cycle, P0 MEFs (SCRC-1040; ATCC) were expanded for at least two passages in treated $10 \mathrm{~cm}$ tissue culture dishes lacking a substrate coating. MEF expansion medium was prepared as follows: add 3.7 $\mathrm{g} / \mathrm{L} \mathrm{NaHCO}_{3}$ (Sigma) to DMEM with high glucose, and supplement with $1 \%$ nonessential amino acids, $1 \%$ sodium pyruvate, $0.1 \mathrm{~mm}$ $\beta$-mercaptoethanol, $1 \%$ penicillin/streptomycin (all from Life Technologies), and $10 \%$ FBS (Atlanta Biologicals) maintained at $37^{\circ} \mathrm{C}$ with $5 \%$ $\mathrm{CO}_{2}$. MEFs were plated at $20,000 \mathrm{cells} / \mathrm{cm}^{2}$ and allowed to incubate for $24 \mathrm{~h}$ before transfection. MEF transfection medium is identical to MEF expansion medium but contains 5\% FBS rather than $10 \%$ and excludes penicillin/streptomycin. Mimic transfection was performed by first incubating RNAiMAX (Life Technologies) with either miR-297c-5p mimic, FAM-labeled negative control mimic or transfection reagent alone (all from Life Technologies) for $30 \mathrm{~min}$ in serum-free Opti-MEM (Life Technologies) at $37^{\circ} \mathrm{C}$ according to the manufacturer protocol. Mimics were added at $10 \mathrm{pmol} / \mathrm{ml}$ to cultures already containing MEF transfection medium and allowed to incubate overnight. An Opti-MEMalone condition was also included to identify effects of transfection reagent alone. The following morning, cells were washed $(2 \times)$ with DPBS to remove serum containing media and harvested by trypsinization for 3 min at $37^{\circ} \mathrm{C}$, after which cells were collected and diluted with MEF media containing $10 \%$ FBS and centrifuged at $300 \times g$ for $5 \mathrm{~min}$. Following centrifugation, cell pellets were washed $(2 \times)$ with DPBS to remove media and trypsin. Finally, the supernatant was aspirated, and ice-cold $70 \%$ ethanol $(\mathrm{EtOH})$ was added to the cell pellets with gentle trituration and allowed to fix for at least $48 \mathrm{~h}$ at $-20^{\circ} \mathrm{C}$. Following fixation, cells were centrifuged at $300 \times g$ for $5 \mathrm{~min}$ and washed $(2 \times)$ with DPBS. Cells were then stained overnight with the DNA intercalator propidium iodide (PI) in PBS containing $600 \mu \mathrm{g} / \mathrm{ml}$ RNase A to assess DNA content. The following day, cells were analyzed with a FACSCaliber Flow Cytometer (Immunocytometry Systems, BD Biosciences). Data were exported as FCS files and further analyzed using Flowjo software (www.treestar. com).

To assess the effect of miR-297c-5p overexpression or CCNT2 knockdown on the oligodendroglial cell cycle, rOPC cultures were transduced with lenti-miR-297c-5p, lenti-CCNT2-shRNA, or control, as stated above. Transduced rOPCs were collected by trypsinization $3 \mathrm{~d}$ after transduction, fixed with cold $70 \% \mathrm{EtOH}$ overnight at $-20^{\circ} \mathrm{C}$, and stained with $300 \mu \mathrm{g} / \mathrm{ml}$ PI in PBS containing $3.8 \mathrm{~mm}$ trisodium citrate, $600 \mu \mathrm{g} / \mathrm{ml}$ RNase A, and $0.1 \%$ Triton X-100 for $1 \mathrm{~h}$ at $37^{\circ} \mathrm{C}$. Cells were analyzed using a BD-LSRII (BD Biosciences) and FlowJo. For all cell cycle experiments, $G_{1} / G_{0}$ and $G_{2} / M$ histogram peaks were fit to the Watson pragmatic model (Watson et al., 1987), and the $G_{1} / G_{0}$, $\mathrm{S}$-phase and $\mathrm{G}_{2} / \mathrm{M}$ frequencies were quantified. Two-way ANOVA was performed with Bonferroni post-test, and data are presented as the mean $\pm \mathrm{SD}$ (MEF cell cycle experiments, $n=5$; rOPC cell cycle experiments, $n=3)$.

\section{Results}

CNPase-EGFP ${ }^{+}$cells sorted from the corpus callosum following cuprizone demyelination and remyelination have an OPC phenotype

Immunohistochemistry (IHC) staining of CNPase-EGFP ${ }^{+}$ brains confirmed that EGFP was specific to oligodendroglia and not to other CNS cell types (Fig. $1 A-C$ ). Confocal microscopy of CNPase-EGFP and NG2 coexpression demonstrates the presence of $\mathrm{CNPase}-\mathrm{EGFP}^{+} / \mathrm{NG}^{+}{ }^{+} \mathrm{OPC}$ within the adult mouse corpus callosum (Fig. 1D). EGFP intensity was used to confirm that cuprizone-induced demyelination and remyelination within the corpus callosum was accompanied by a reduction and return of callosal CNPase-EGFP expression, respectively. EGFP intensity within the callosal ROI was significantly decreased at 6 weeks of cuprizone treatment compared with control $(p<0.05)$ and was not significantly different from control at 6 weeks of recovery (Fig. 1E-H).

FACS analysis showed a bright cell population of CNPase$\mathrm{EGFP}^{+}$OPCs (gate R3; Fig. 2B) that was consistently present above background established with WT corpus callosum (gate R1; Fig. 2A). For all samples, $\mathrm{EGFP}^{+}$cells accounted for $52.34 \pm$ $7.45 \%$ of total events (gate R3/R1; Fig. $2 B$ ). To confirm the phenotype of CNPase-EGFP ${ }^{+}$cells, gene expression levels of sorted populations from mice treated for 6 weeks of cuprizone and controls were assessed with qRT-PCR for the following genes: OPCs (Olig2 and PDFGR $\alpha$ ), OLs (Olig2, PLP, and MBP), astrocytes (GFAP), and neurons (Map2; Fig. 2E). When compared with RNA isolated from an equal postdissection volume of whole callosal tissue (which contains RNA from all CNS cell types), expression profiles were highly consistent with an OPC phenotype (Fig. $2 E)$. Sorted cells displayed robust expression levels of Olig2 and PDGFR $\alpha$, highly expressed genes in OPCs (Fig. 2E). PLP and MBP (two genes specifically expressed in myelinating OLs) were not highly expressed in sorted control cells, suggesting an absence of mature OLs from sorted populations (Fig. 2E). This is not surprising as mature OLs are more susceptible to death than OPCs during the isolation protocol. Interestingly, PLP expression was significantly higher in OPCs treated for 6 weeks with cuprizone versus controls (Fig. 2E). PLP mRNA expression has been previously described in premyelinating $\mathrm{NG}_{2}{ }^{+} \mathrm{OPC}$ during development (Mallon et al., 2002; Ye et al., 2003), thus suggesting that during cuprizone remyelination, CNPase-EGFP ${ }^{+}$OPCs represent a more mature population of OPCs compared with the naive corpus callosum. Genes representative of astrocytes (GFAP) and neurons (Map2) displayed low expression levels compared with whole corpus callosum, suggesting that there is little contamination from these cell types in the sorted population. CNPase has previously been suggested to be expressed in activated microglia (Wu et al., 2006; Yang et al., 2014). To confirm that sorted CNPase-EGFP ${ }^{+}$populations did not contain cells of a myeloid lineage, anti-CD45-perCP was used to identify the proportion of $\mathrm{CD}_{4} 5^{+} / \mathrm{CNPase}-\mathrm{EGFP}{ }^{+}$cells. No $\mathrm{CD} 45^{+}$cells were present when sorting with the IgG-perCP isotype control (gate R4; Fig. 2D). Although CD45-perCP ${ }^{+}$cells were present, only a negligible proportion was $\mathrm{CD} 45^{+} / \mathrm{CNPase}^{-\mathrm{EGFP}^{+}}$representing $<0.2 \%$ of all CNPase-EGFP ${ }^{+}$events (gate R4; Fig. 2C). These data suggest that sorted callosal CNPase-EGFP ${ }^{+}$cells represent a highly enriched population of OPCs in both cuprizone-treated and control brains. 

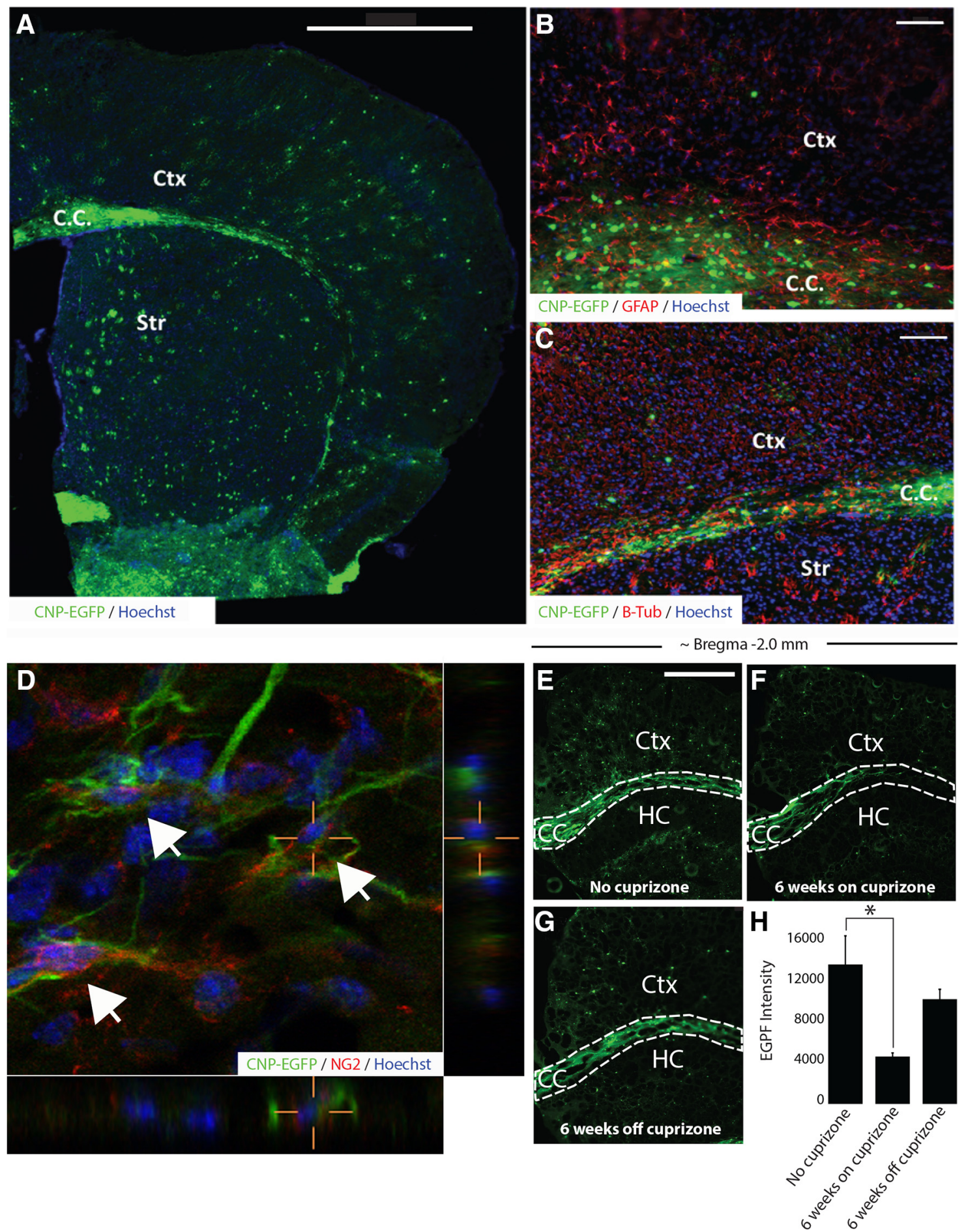

Figure 1. Oligodendroglia-specific CNPase-EGFP reporter expression decreases during cuprizone-induced demyelination and returns during remyelination. $A-C$, CNPase-EGFP is expressed in oligodendroglial lineage cells throughout the cortex (Ctx), striatum (Str), and corpus callosum (CC) with no coexpression with GFAP ${ }^{+}$astrocytes or $\beta$-tubulin-positive ( $\beta$-Tub ${ }^{+}$) neurons. $\boldsymbol{D}$, Confocal microscopy within the adult mouse CC demonstrates the coexpression of CNPase-EGFP in NG2 ${ }^{+} \mathrm{OPCs}$ (highlighted by the white arrows). The crosshairs correspond to the orthogonal slice for one example of a CNPase-EGFP ${ }^{+} / \mathrm{NG}^{+} \mathrm{OPC}$. E-H, Following 6 weeks of cuprizone treatment, CNPase-EGFP expression is diminished within the $\mathrm{CC}$ compared with no cuprizone control (demyelination) but recovers 6 weeks after removal of cuprizone (remyelination). Data in $\boldsymbol{H}$ are reported as the mean \pm SD ( $n=3 /$ group). ${ }^{*} p<0.05 . S$ cale bars: $\boldsymbol{A}, 1500 \mu \mathrm{m} ; \boldsymbol{B}, \boldsymbol{C}, 100 \mu \mathrm{m} ; \boldsymbol{E}-\boldsymbol{G}$, $1000 \mu \mathrm{m}$.

Sfmbt 2 cluster miRNAs share similar expression profiles during cuprizone-induced demyelination and remyelination Of 1215 mouse miRNAs assayed in the microarray, 325 were identified as being present in each sample and three-dimensional PCA showed good clustering of samples within each group (data not shown). Group ANOVA revealed that 252 miRNAs changed significantly across control and cuprizone treatment conditions. No differences were identified between the 8 and 20 week nocuprizone groups. Therefore, the 8 week control group was used for all fold change comparisons, and no miRNAs were excluded on the basis of age-dependent expression. We compared log2 fold change values for each experimental condition versus control (no 
cuprizone) of the 97 most significant miRNAs (top $40 \%$ organized by $p$ value) identified via array (group ANOVA with a Benjamini-Hochberg FDR correction). Dendrogram analysis demonstrated that samples within a particular cuprizone treatment condition were highly consistent (Fig. 3A). Hierarchical clustering analysis revealed a cluster of 51 miRNAs all sharing similar expression patterns (fold change vs control) with the lowest values occurring at 6 weeks of cuprizone treatment (Fig. $3 A, B$ ). Thirty-eight miRNAs within this cluster $(73 \%)$ are members of the Sfmbt 2 miRNA family (Fig. 3B, identified with ${ }^{*}$ ), a rodent-specific cluster of 65 miRNAs located inside a $54 \mathrm{~kb}$ region within intron 10 of the polycomb group gene Sfmbt2 (Wang et al., 2011). Several Sfmbt 2 miRNAs are represented multiple times within the cluster, many of which display high target sequence similarity with other Sfmbt 2 cluster members (some with near perfect alignment; Table 1). Specifically, miRs-297a, -297b, $-297 \mathrm{c},-466 \mathrm{~b},-467 \mathrm{a}$, and $-669 \mathrm{a}$ are the most represented members of the cluster (Table 1). Expression patterns of all Sfmbt 2 miRNAs are similar across cuprizone treatment conditions (Fig. 3B). Post hoc tests revealed that at 3 weeks of cuprizone treatment, no Sfmbt 2 miRNAs were significantly different from control while a significant downregulation was identified at 6 weeks of cuprizone treatment (Fig. 3B). At 3 and 6 weeks of recovery, expression values approached control levels, with no Sfmbt 2 miRNAs being significantly different from control at 6 weeks of recovery (Fig. $3 B$ ). Target prediction (miRanda) of Sfmbt 2 miRNAs listed in Table 1 generated a final list of 463 genes for which disease and biofunction analysis revealed enrichment for nervous system development, cellular movement, cellular growth and proliferation, cell cycle, proliferation, and migration of neuroglia/ brain cells (Table 2). Pathway analysis revealed enrichment for axonal guidance, neuregulin, PDGF, FGF, glioma, and EGF signaling pathways (Table 2). These data suggest that Sfmbt2 miRNAs display a collective decrease in expression at 6 weeks of cuprizone treatment and are predicted to repress genes involved in OPC proliferation pathways.

miR-297c expression accompanies both mOPC differentiation in vitro and white matter development in vivo We assessed the miRNA expression of several Sfmbt 2 miRNAs to identify which could potentially regulate oligodendroglial function in isolated $\mathrm{O}^{+}{ }^{+}$mOPCs with qRT-PCR following $72 \mathrm{~h}$ of proliferation (Fig. 4A) or differentiation (Fig. 4B). IHC analysis
A

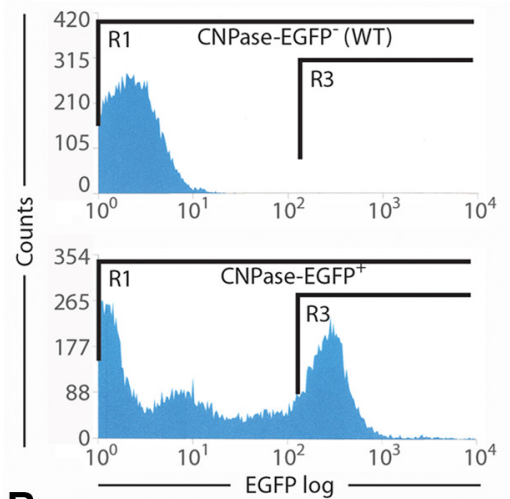

B



C

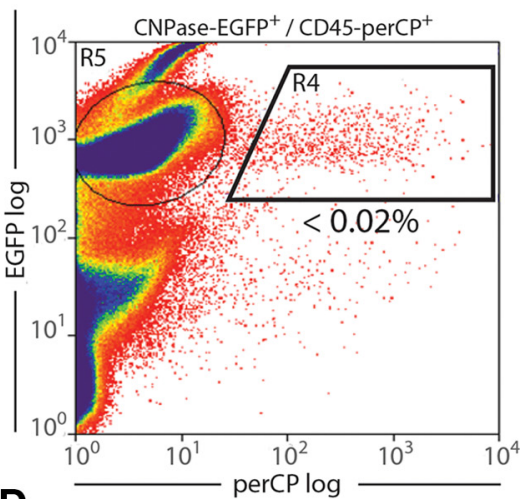

D



\section{E Phenotypic Marker Gene Profile of CNPase ${ }^{+}$OPCs (Normalized to GAPDH)}

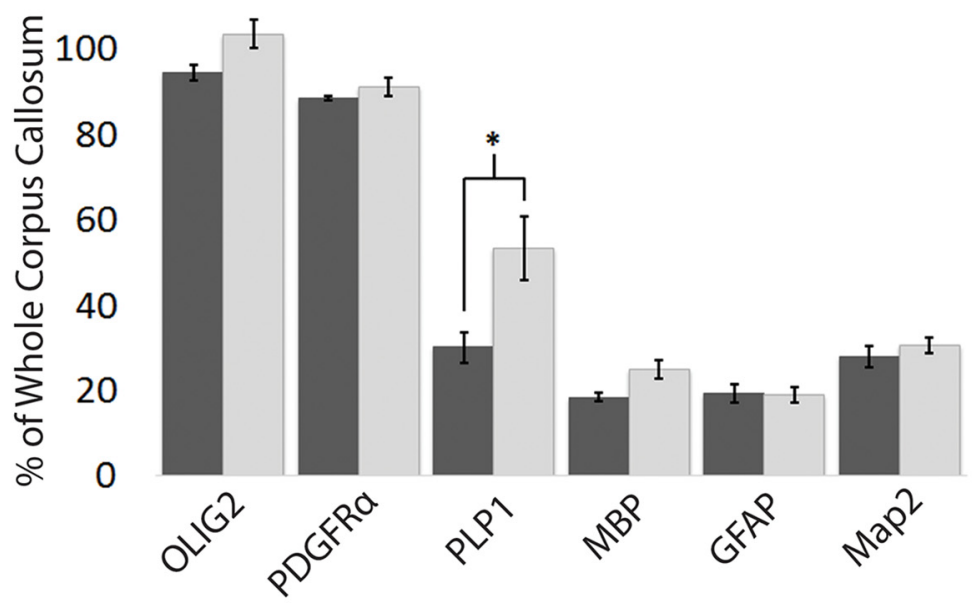

No Cuprizone

6 Weeks Cuprizone

Figure 2. Sorted callosal CNPase-EGFP ${ }^{+}$cells express mRNA transcripts consistent with an OPC phenotype with little contamination from other CNS cell types. $\boldsymbol{A}$, Histograms of EGFP ${ }^{+}$cell counts from WT and CNPase-EGFP ${ }^{+}$mice. $\boldsymbol{B}-\boldsymbol{D}$, FACS plots of CNPase-EGFP ${ }^{+}$, CNPase-EGFP $^{+} / \mathrm{lgG}$-perCP ${ }^{+}$, and CNPase-EGFP ${ }^{+} / \mathrm{CD}^{2} 4$-perCP ${ }^{+}$sorts. $E$, qRT-PCR data of phenotypic gene markers vs whole CC. Data in $E$ are reported as the mean \pm SD ( $n=3 /$ group). ${ }^{*} p<0.05$.

confirmed appropriate differentiation conditions. At $72 \mathrm{~h}$ in proliferation condition, $93.94 \pm 6.84 \%$ of total Hoechst ${ }^{+}$nuclei were also $\mathrm{A} 2 \mathrm{~B} 5^{+}$, and $83.24 \pm 9.35 \%$ were $^{+} 4^{+}$(Fig. $4 A, C$ ). Following $72 \mathrm{~h}$ of differentiation in media lacking mitogenic growth factors and containing $\mathrm{T} 3$, process elongation and arborization were accompanied by a reduced percentage of A2B5 ${ }^{+}$ cells to $14.87 \pm 7.77 \%$ and $\mathrm{O} 4{ }^{+}$cells $46.23 \pm 7.77 \%$ relative to 

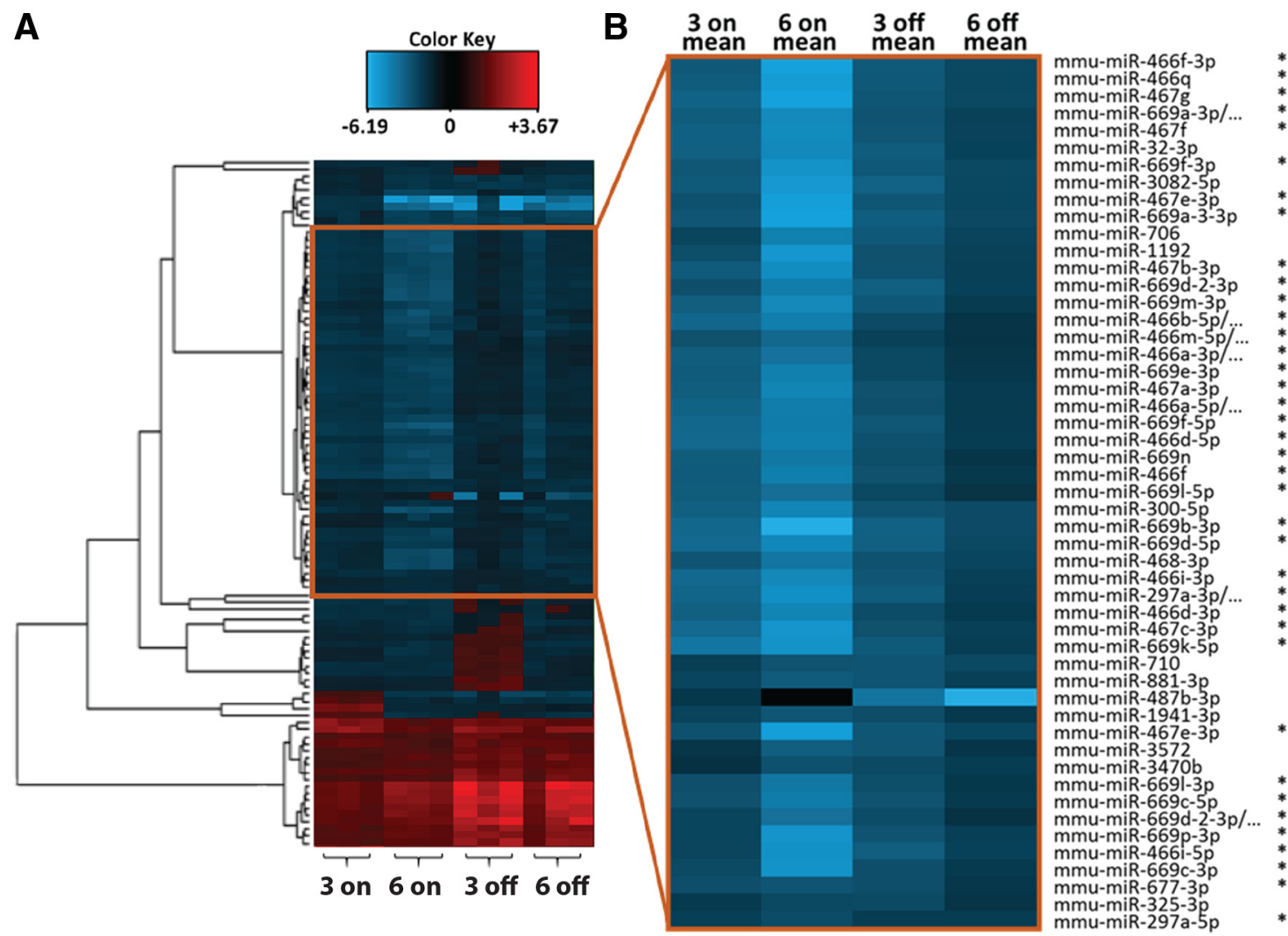

Figure 3. miRNA microarray expression patterns in callosally derived CNPase-EGFP ${ }^{+}$cells after a cuprizone diet for 3 and 6 weeks or recovery for 3 and 6 weeks. A, Heat map displaying log 2 fold change (FC) vs control (no cuprizone) for the top 40\% (97) most significant miRNAs (FDR 10\%) identified via array. B, Hierarchical clustering of differentially expressed miRNAs identified a cluster of miRNAs sharing similar expression patterns, with the lowest values occurring at 6 weeks of cuprizone treatment. Heat map represents the mean FC vs control for each group. Thirty-eight of the clustered miRNAs (73\%) are members of the Sfmbt2-miRNA family (highlighted with an *). Red and blue values indicate positive and negative fold change values, respectively.

Table 2. Functional enrichment of Sfmbt2 miRNA target genes

\begin{tabular}{|c|c|c|c|c|c|}
\hline Diseases and biofunctions & No. of genes (of 463) & $p$ value (IPA) & Signaling pathways & No. of genes (of 463) & $p$ value (IPA) \\
\hline Nervous system development & 78 & 4.15E-03-1.41E-06 & Axonal guidance & 22 & $1.15 \mathrm{E}-04$ \\
\hline Cellular movement & 77 & 4.15E-03-1.81E-04 & Neuregulin & 7 & $2.40 \mathrm{E}-03$ \\
\hline Cellular growth and proliferation & 65 & 4.10E-03-1.14E-04 & PDGF & 5 & $2.22 \mathrm{E}-02$ \\
\hline Cell cycle & 59 & 4.15E-03-5.83E-05 & FGF & 5 & $3.23 \mathrm{E}-02$ \\
\hline Proliferation & 50 & 4.05E-03-2.96E-04 & Glioma & 5 & $4.83 \mathrm{E}-02$ \\
\hline Migration of neuroglia and brain cells & 11 & $3.35 \mathrm{E}-03-1.81 \mathrm{E}-04$ & EGF & 4 & $2.91 \mathrm{E}-02$ \\
\hline
\end{tabular}

Functional enrichment was performed using ingenuity pathway analysis (IPA) of the common target genes of Sfmbt2 miRNAs identified in Table 1. Target prediction was performed with miRanda and only targets with a mirSVR score less than -1.3 were considered, resulting in a final list of 463 common targets. Lower mirSVR scores correspond to a stronger predicted miRNA-target interaction. Enriched disease and biofunctions were consistent with cellular activities commonly associated with remyelinating OPCs, such as migration and proliferation. Signaling pathways known to be involved in proliferating and migrating OPCs were also enriched.

total Hoechst ${ }^{+}$nuclei (Fig. $4 B, C$ ). Moreover, $\mathrm{O}^{+}$cells, which were not present in proliferation conditions, were identified following $72 \mathrm{~h}$ differentiation constituting $41.88 \pm 18.64 \%$ of total Hoechst $^{+}$nuclei (Fig. 4B,C). GFAP ${ }^{+}$immunoreactivity following $72 \mathrm{~h}$ of proliferation was $1.54 \pm 0.05 \%$ of total Hoechst ${ }^{+}$ nuclei (Fig. $4 A, C$ ) and increased to $27.50 \pm 8.83 \%$ of total Hoechst $^{+}$nuclei following $72 \mathrm{~h}$ of differentiation (Fig. $4 \mathrm{~B}, \mathrm{C}$ ). Of miRNAs screened with qRT-PCR, one was found to be consistently upregulated during primary OPC differentiation versus proliferation: mmu-miR-297c-5p. Greater expression of miR297c-5p consistently accompanied differentiation with $3.18 \pm$ 0.71 -fold higher values than for proliferating OPCs $(p<0.05$; Fig. $4 D)$. As a positive control for differentiation, mmu-miR338-5p was also included as it is an oligodendrocyte-specific miRNA that is well known to increase concomitant with OPC differentiation in vitro (Dugas et al., 2010; Zhao et al., 2010). Following differentiation, miR-338-5p values were $1.74 \pm 0.03$ fold greater than proliferating mOPCs ( $p<0.05$; Fig. $4 D)$.
To determine whether miR-297c-5p levels are regulated during myelin development, we isolated RNA from dissected corpus callosum at 1,2, and 3 weeks of postnatal development, coinciding with early to vigorous myelin development. We detected a significant increase in miR-297c-5p levels at 2 weeks compared with 1 week by qRT-PCR (Fig. 5A). miR-297c-5p levels returned to baseline at 3 weeks (Fig. 5A). Levels of miR-338-5p, a known regulator of oligodendrocyte development in vivo (Lau et al., 2008; Zhao et al., 2010), were significantly increased at 2 and 3 weeks of postnatal development (Fig. $5 B$ ). These data suggest that miR-297c-5p may also play a role in the development of the oligodendroglial lineage in vivo.

\section{miR-297c regulates cell cycle and differentiation}

To identify miR-297c-5p as a regulator of cell cycle, we ectopically expressed miR-297c-5p via mimic transfection into proliferating MEFs. Following transfection, MEF cell cycle $G_{1} / G_{0}$ frequencies were significantly higher than in control transfected 

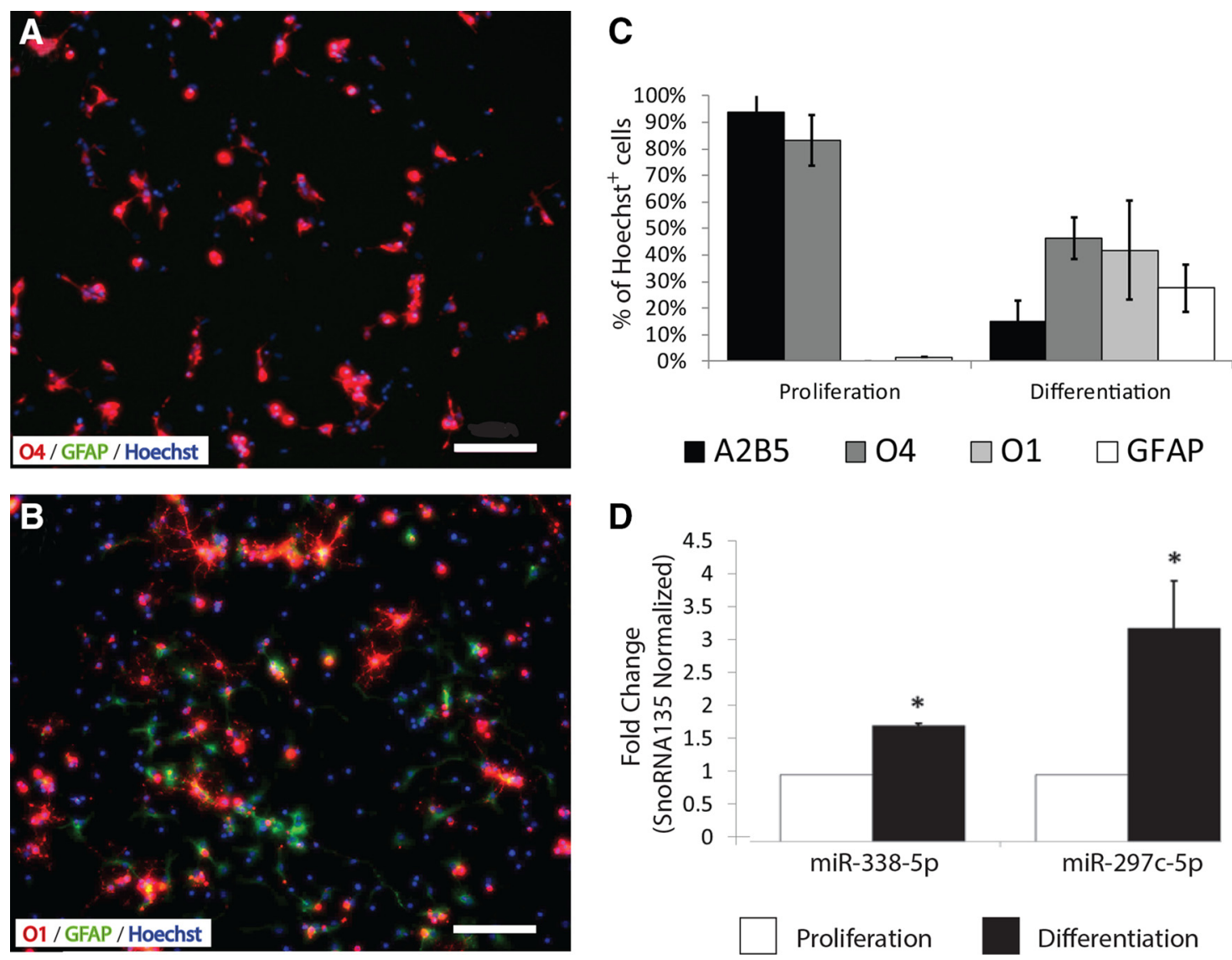

D

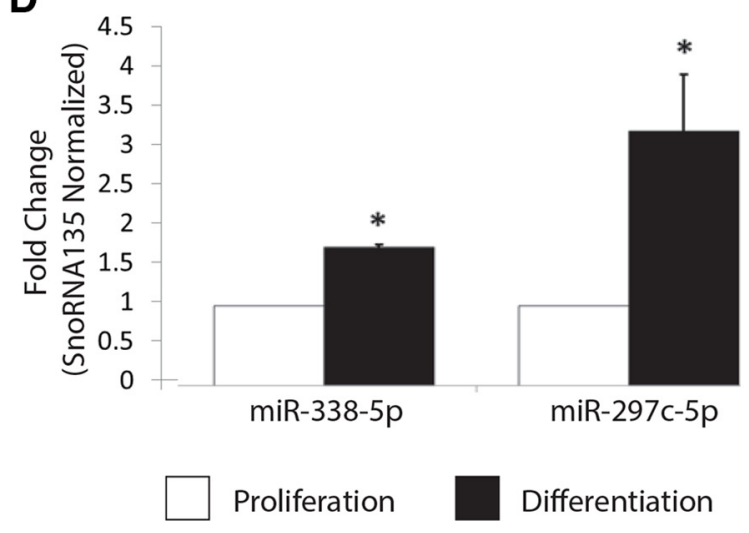

Figure 4. Increased miR-297c-5p levels during OPC differentiation. $A$, ICC of proliferating mOPCs treated with FGF2 and PDGFR $\alpha$ (04/GFAP/Hoechst). B, ICC of differentiating mOPCs lacking mitogens plus T3 (01/GFAP/Hoechst). C, Quantification of ICC data (counts/Hoechst ${ }^{+}$cells). D, qRT-PCR of miR-338-5p and miR-297c-5p in proliferating and differentiating m0PCs normalized to SnoRNA135. Data in $C$ and $\boldsymbol{D}$ are reported as the mean \pm SD $(\boldsymbol{C}, n=3 ; \boldsymbol{D}, n=4) .{ }^{*} p<0.05$. Scale bars: $\boldsymbol{A}, \boldsymbol{B}, 100 \mu \mathrm{m}$.

MEFs (Fig. 6A). To determine the function of miR-297c-5p in oligodendrocytes, rOPC cultures were transduced with a lentivirus-expressing miR-297c. Cell cycle analysis of the transduced rOPC cultures revealed a significant increase in the $\mathrm{G}_{1} / \mathrm{G}_{0}$ frequency of cultures transduced with the miR-297c-5p lentivirus (Fig. 6B). Together, the MEF and rOPC cell cycle analyses suggest disruption of the $G_{1} / G_{0}$ to S-phase transition.

To assess the role of miR-297c-5p in oligodendrocyte differentiation, we tested whether OPC differentiation was affected by overexpression of miR-297c-5p. rOPC cultures were differentiated for $3 \mathrm{~d}$ after transduction with miR-297c-5p lentivirus and stained for markers of OL differentiation as well as GFAP to identify astrocytes. No appreciable GFAP staining was observed in cultures transduced with control or miR-297c-5p lentivirus (data not shown). A significant increase in $\mathrm{O}^{+}$cells was detected in cultures transduced with miR-297c-5p lentivirus (Fig. 6C-E), suggesting that miR-297c-5p promotes oligodendrocyte differentiation.

miR-297c functionally targets the cyclin T2 (CCNT2) 3'UTR To identify the mechanism by which miR-297c-5p regulates OPCs, we tested whether it binds within the $3^{\prime}$ UTR of its pre-
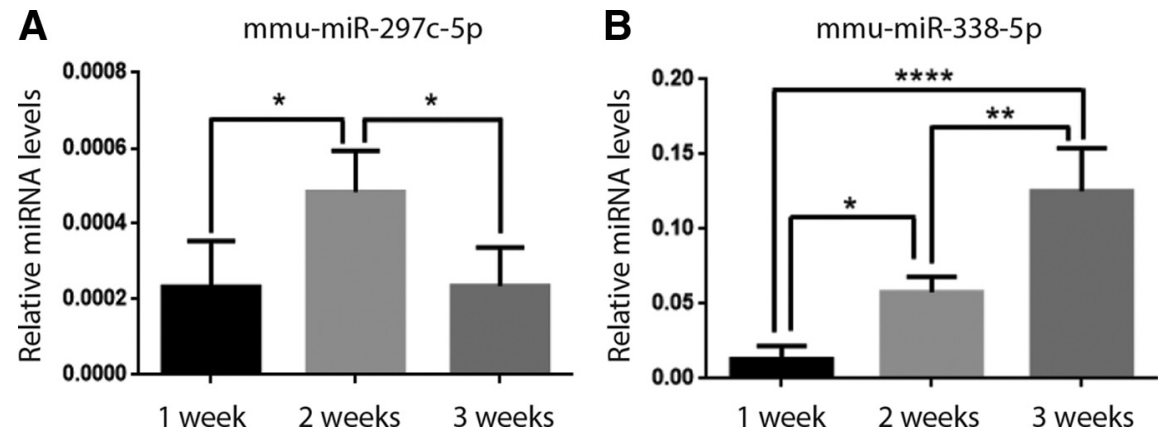

Figure 5. Increased miR-297c-5p levels during oligodendroglial development. $A, B$, Total RNA was isolated from corpus callosum of wild-type mice at 1, 2, and 3 weeks of age, and levels of mir-297c-5p and miR-338-5p were measured by qRT-PCR and normalized to U6 snRNA $(n=4)$. Data are reported as the mean \pm SD. ${ }^{*} p<0.05,{ }^{* *} p<0.01,{ }^{* *} p<0.001$.

dicted target gene, CCNT2 (Fig. 7A). HEK 293FT cells were cotransfected with renilla luciferase, control or mmu-miR-297c mimic, and either an empty vector, CCNT2 3 'UTR-containing, or mutant CCNT2 3'UTR-containing firefly luciferase reporter. After $24 \mathrm{~h}$, luciferase activity was measured in lysates, and firefly luciferase levels were normalized to renilla luciferase levels. Luciferase activity from reporters not containing the CCNT2 $3^{\prime}$ UTR were unaffected by the presence of the mmu-miR-297c mimic. Activity of the CCNT2 3'UTR-containing reporter in the presence of mmu-miR-297c mimic was $29 \pm 15 \%$ of the activity seen when control mimic was present (Fig. $7 B$ ). To determine whether this effect was due to targeting of the identified site from 

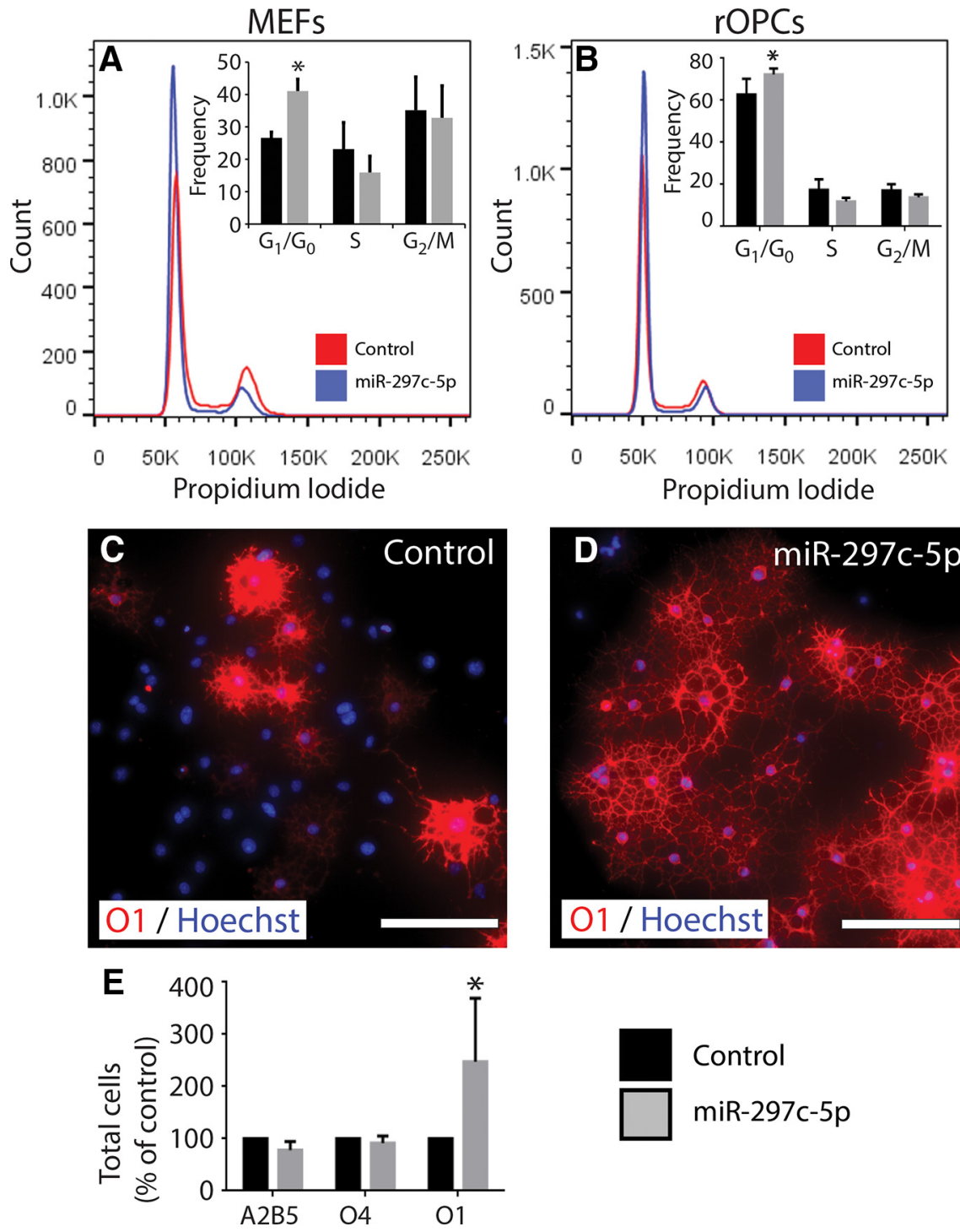

Figure 6. miR-297c-5p blocks cell cycle progression in MEFs and rOPCs, and promotes oligodendrocyte differentiation. $A$, MEFs transfected with miR-297c-5p mimics displayed greater $G_{1} / G_{0}$ frequencies compared with scrambled miRNA control mimics $(n=$ 5). $\boldsymbol{B}$, rOPCs transduced with a lentivirus-expressing miR-297c-5p display greater $G_{1} / G_{0}$ frequencies compared with scrambled miRNA control $(n=3)$. $C-E$, Cell counts of differentiating rOPCs transduced with miR-297c-5p lentivirus demonstrated a greater proportion of $01^{+} /$Hoechst ${ }^{+}$cells compared with control $(n=3)$. Data are reported as the mean \pm SD. ${ }^{*} p<0.05$. Scale bars: C, D, $100 \mu \mathrm{m}$.

Figure $7 A$, site-directed mutagenesis was performed to alter three nucleotides within the sequence complimentary to the miR297c-5p seed region (Fig. 7A). Activity of the CCNT2 3'UTR mutant-containing reporter in the presence of mmu-miR-297c mimic was $54 \pm 13 \%$ of the activity seen when control mimic was present (Fig. $7 B$ ), but this was not significant from either the full-length 3'UTR or empty vector groups. These data suggest that miR-297c-5p targets the CCNT2 3'UTR, primarily through the identified target sequence.

To confirm that the effect of miR-297c-5p overexpression on rOPC differentiation was mediated by its interaction with CCNT2, rOPCs were transduced with two different shRNAs targeting CCNT2 or control shRNA for $24 \mathrm{~h}$ before differentiation (Fig. $7 C-F$ ). After $72 \mathrm{~h}$ of differentiation, a significant increase in $\mathrm{O}^{+}{ }^{+}$staining was observed in cultures transduced either with CCNT2 shRNA (CCNT2 shRNA1 and CCNT2 shRNA2; Fig. $7 D-F$ ) or with control (Fig. $7 C, F$ ). However, transduction of either CCNT2 shRNA1 or CCNT2 shRNA2 had no effect on cell cycle (data not shown). These data suggest that, while miR-297c-5p disrupts cell cycle progression and promotes differentiation of rOPCs, the mechanisms (and gene targets) by which it does so are distinct.

\section{Discussion}

$\mathrm{CNPase-EGFP}^{+}$cells were phenotypic of OPCs (Fig. 1) and when sorted from the corpora callosa of cuprizone-treated mice (Fig. 2), displayed dynamic miRNA expression patterns accompanying demyelination and remyelination compared with control (Fig. 3). Microarray analysis identified a cluster of miRNAs that decrease at 6 weeks of cuprizone treatment and progressively return to control levels at 3 and 6 weeks of recovery (Fig. 3). The process of cuprizone remyelination is complex, involving an initial OPC propagation phase and a subsequent maturation phase (Kipp et al., 2009). Although there is some overlap between these two events, previous studies have shown that during the 6 weeks on-6 weeks off cuprizone treatment paradigm, the peak of OPC proliferation begins by 5-6 weeks despite ongoing cuprizone treatment (Mason et al., 2001; Gudi et al., 2014). Following the cessation of cuprizone treatment, the peak of oligodendrocyte maturation occurs at 2-3 weeks post-cuprizone treatment with remyelination resolving by 6 weeks of recovery (Morell et al., 1998; Jurevics et al., 2001). The decreased expression of Sfmbt 2 miRNAs at 6 weeks of cuprizone treatment and their returning expression (relative to control) at 3 and 6 weeks off cuprizone treatment suggest involvement in the proliferation and maturation phases of remyelination, respectively.

Although OPCs retain mitotic competence well past developmental gliogenesis (ffrench-Constant and Raff, 1986; Wolswijk and Noble, 1989), robust OPC proliferation occurs only in the presence of demyelination-induced cues (Keirstead et al., 1998; Redwine and Armstrong, 1998). In the context of the cuprizone model, the callosal environment at 6 weeks of cuprizone treatment contains high levels of the growth factor FGF2, a potent OPC mitogen (Mason et al., 2000; Hibbits et al., 2012). Considering the canonical role for miRNAs in translational repression of targeted transcripts, the decreased expression of Sfmbt 2 miRNAs at 6 weeks of cuprizone treatment (a time point when callosal growth factors are highest) suggests that these miRNAs normally prevent proliferation by repressing the expression of genes involved in mitogenic signaling pathways. However, during demyelination-induced OPC proliferation, Sfmbt2 miRNA levels decrease, thereby de-repressing translation of their targets and thus promoting proliferation. Past studies have shown that clustered miRNAs often share similar expression patterns (Baskerville and Bartel, 2005; Chan et al., 2012), and we hypothesized 
that Sfmbt 2 miRNAs also adhere to this principle. Together, the coexpression patterns of Sfmbt 2 miRNAs and high sequence similarity to other family members (Table 1) suggest that they may act in concert to repress genes that regulate similar biological functions and signaling pathways. Functional enrichment of genes predicted as common targets of miRNAs listed in Table 1 (Table 2) demonstrate that the predicted target gene signature of highly represented Sfmbt2 miRNAs is not only consistent with cellular proliferation in general (i.e., cell cycle, cellular growth, and proliferation), but more specifically with OPC proliferation (i.e., PDGF, FGF, neuregulin, and glioma pathways).

To more directly assess the role of Sfmbt 2 miRNAs in OPCs, we compared expression levels of selected Sfmbt2 miRNAs in proliferating versus differentiating primary mOPCs in vitro and found miR-297c-5p to be consistently upregulated during differentiation (Fig. 4). Moreover, when ectopically expressed in both MEFs and rOPCs, miR-297c-5pinduced cell cycle arrest (Fig. 6A,B). In MEFs, miR-297c-5p is not normally expressed and thus provided a platform to assess its functional role with no interference from endogenous expression (Houbaviy et al., 2003), while rOPCs provided functional assessment in the context of oligodendroglia. While cell cycle arrest alone is not sufficient to induce OL differentiation, it is a necessary prerequisite for the transition to a mature phenotype (Casaccia-Bonnefil et al., 1997; Durand et al., 1998; Tang et al., 1999; CasacciaBonnefil and Liu, 2003). Thus, we also tested the possibility that miR-297c-5p regulates OL development in vivo and differentiation in vitro. To that end, we assessed the expression levels of callosal miR-297c-5p and -338-5p (a known regulator of oligodendrocyte development and differentiation; Zhao et al., 2010) throughout early mouse development. Both increased in expression from 1 to 2 weeks (Fig. 5), but only miR-338-5p levels continued to increase as miR-297c-5p levels were no different from control by 3 weeks (Fig. $5 B$ ). The difference at 3 weeks suggests that miR-297c-5p may only regulate the transition from OPC to early OL and its levels decrease as myelination completes by 3 weeks. However, miR-338-5p levels continue to increase as callosal OLs begin to outnumber OPCs, which is not surprising considering its robust expression in differentiating OLs (Dugas et al., 2010; Zhao et al., 2010; Birch et al., 2014). To confirm a role for miR-297c-5p during the OPC-to-OL transition in a cellspecific manner, we overexpressed miR-297c-5p in differentiating rOPCs and found that it increased the proportion of cells expressing O1, a marker of OLs (Fig. 6C-E). These data suggest


Figure 7. miR-297c-5p targets the CCNT2 3'UTR to promote oligodendrocyte differentiation. $\boldsymbol{A}$, Top, Alignment showing base pairing of the miR-297c-5p seed region and CNT2 3' UTR. Bottom, Alignment displaying the mutation introduced into the CCNT2 $3^{\prime}$ UTR sequence complimentary to the miR-297c-5p seed region. $B$, HEK-293FT cells were cotransfected with a renilla luciferase internal control vector and a firefly luciferase vector containing no 3' UTR, full-length CCNT2 3' UTR, or full-length CCNT2 3' UTR with mutated miR-297c-5p seed region and either control or miR-297c-5p mimic. Firefly luciferase levels normalized to renilla luciferase levels in miR-297c-5p mimic transfected cells were quantified as a percentage of levels in control mimic transfected cells $(n=3)$. Data are reported as the mean \pm SD. ${ }^{*} p<0.05$. $C-F$, Cell counts of differentiating rOPCs transduced with lentivirusexpressing shRNA targeting CCNT2 demonstrated a greater proportion of $01^{+} /$Hoechst ${ }^{+}$cells compared with control $(n=3)$. Data are reported as the mean \pm SD. ${ }^{*} p<0.05$. Scale bars: $\boldsymbol{C}-\boldsymbol{E}, 50 \mu \mathrm{m}$.

that miR-297c-5p has a dual role in repressing OPC proliferation and promoting OL maturation.

To understand the mechanism by which miR-297c-5p impacts in vitro proliferation and differentiation as well as in vivo development, target prediction was performed using three separate algorithms (microRNA.org, miRDB.org, and targetscan.org) that each predicted an interaction between miR-297c-5p and CCNT2, the regulatory subunit of the P-TEFb complex (Peng et al., 1998). Moreover, this interaction is also conserved in humans. Interestingly, several other Sfmbt 2 miRNAs are also predicted to target loci within the mouse CCNT2 3'UTR, a likely consequence of the high seed sequence homology among Sfmbt2 
miRNA family members (Table 1 ). The binding of miR-297c-5p to the target site within the CCNT2 3'UTR was confirmed with a luciferase reporter assay (Fig. 7A,B). Unexpectedly, there was also moderate knockdown observed for the mutated target site (Fig. 7B). This suggests that additional CCNT2 target sites may exist and/or that miR-297c-5p targeting may be partially determined by non-seed sequence binding rather than seed sequence complementarity alone. Although the seed sequence is the most evolutionarily conserved miRNA region (Lewis et al., 2003) and is commonly regarded as the most important to mRNA targeting (Bartel, 2004), the contribution of non-seed sequence binding has recently received more consideration (Bartel, 2004). Sequence pairing outside of the seed region can both supplement seed-target site recognition and compensate for seed mismatches in cases where matches are present between miRNA nucleotides 13 and 16, and the complimentary 3'UTR region (Grimson et al., 2007; Bartel, 2009). Both the full-length and mutated $3^{\prime}$ UTR sequences we describe here have two matches within the 13-16 nt miRNA region (Fig. 7A).

To confirm that CCNT2 is a functional target of miR-297c-5p, we transduced rOPCs with two different CCNT2-specific shRNAs to assess the effects on cell cycle and differentiation. While cell cycle was not altered, transduction of CCNT2 shRNA1 and shRNA2 both enhanced differentiation compared with control (Fig. 7C-F). Together, these data suggest that miR-297c-5p regulates both oligodendrocyte cell cycle exit and the initiation of differentiation. However, these effects occur via distinct mechanisms as only differentiation was affected in a CCNT2-dependent manner. This supports previous work demonstrating that pharmacological inhibition of P-TEFb rescued impaired OL differentiation and MBP expression in developing myelin-deficient zebrafish (Kim et al., 2012). Moreover, the CCNT2-independent effect of miR-297c-5p overexpression on cell cycle arrest is not unexpected as target prediction analysis reveals a number of genes with known roles in proliferation, including the cell cycle regulators cell cycle progression 1 (CCPG1), cell division cycle 23 (CDC23), and the Wnt signaling pathway-associated frizzled 1 (FZD1), each of which were predicted to be targeted by miR$297 c-5 p$ by at least two algorithms. These data are in support of distinct mechanisms for miR-297c-5p in differentiation and proliferation, and suggest that miR-297c-5p may provide a transitional link between the two states (i.e., proliferation to differentiation).

Together, the decreased OPC expression values (array) at 6 weeks of cuprizone treatment, GO, mOPC differentiation assays, in vivo development analysis, rOPC overexpression experiments, cell cycle assays, and confirmation of a miR-297c-5p-CCNT2 functional interaction provide strong evidence that miR-297c-5p is a regulator of OPC differentiation. It is unclear what cues drive miR-297c-5p and/or the expression of the entire Sfmbt2 cluster. Identifying the specific factors responsible for its transcription and the extracellular and intracellular signaling events involved is crucial to developing a complete understanding of the biological activity of miR-297c-5p in OPCs. However, its ability to induce OPC differentiation makes it an intriguing target with potential therapeutic relevance, considering the restricted differentiation of OPCs within demyelinating lesions (Goldman and Osorio, 2014). Therefore, efforts to understand the role of miR-297c-5p in pathological conditions and its potential to facilitate repair may provide future clinical value.

\section{References}

Barca-Mayo O, Lu QR (2012) Fine-tuning oligodendrocyte development by microRNAs. Front Neurosci 6:13. CrossRef Medline

Bartel DP (2004) MicroRNAs: genomics, biogenesis, mechanism, and function. Cell 116:281-297. CrossRef Medline

Bartel DP (2009) MicroRNAs: target recognition and regulatory functions. Cell 136:215-233. CrossRef Medline

Baskerville S, Bartel DP (2005) Microarray profiling of microRNAs reveals frequent coexpression with neighboring miRNAs and host genes. RNA 11:241-247. CrossRef Medline

Bettencourt-Dias M, Giet R, Sinka R, Mazumdar A, Lock WG, Balloux F, Zafiropoulos PJ, Yamaguchi S, Winter S, Carthew RW, Cooper M, Jones D, Frenz L, Glover DM (2004) Genome-wide survey of protein kinases required for cell cycle progression. Nature 432:980-987. CrossRef Medline

Birch D, Britt BC, Dukes SC, Kessler JA, Dizon ML (2014) MicroRNAs participate in the murine oligodendroglial response to perinatal hypoxiaischemia. Pediatr Res 76:334-340. CrossRef Medline

Budde H, Schmitt S, Fitzner D, Opitz L, Salinas-Riester G, Simons M (2010) Control of oligodendroglial cell number by the miR-17-92 cluster. Development 137:2127-2132. CrossRef Medline

Cao Q, He Q, Wang Y, Cheng X, Howard RM, Zhang Y, DeVries WH, Shields CB, Magnuson DS, Xu XM, Kim DH, Whittemore SR (2010) Transplantation of ciliary neurotrophic factor-expressing adult oligodendrocyte precursor cells promotes remyelination and functional recovery after spinal cord injury. J Neurosci 30:2989-3001. CrossRef Medline

Casaccia-Bonnefil P, Liu A (2003) Relationship between cell cycle molecules and onset of oligodendrocyte differentiation. J Neurosci Res 72:1-11. CrossRef Medline

Casaccia-Bonnefil P, Tikoo R, Kiyokawa H, Friedrich V Jr, Chao MV, Koff A (1997) Oligodendrocyte precursor differentiation is perturbed in the absence of the cyclin-dependent kinase inhibitor p27Kip1. Genes Dev 11: 2335-2346. CrossRef Medline

Chan WC, Ho MR, Li SC, Tsai KW, Lai CH, Hsu CN, Lin WC (2012) MetaMirClust: discovery of miRNA cluster patterns using a data-mining approach. Genomics 100:141-148. CrossRef Medline

De Luca A, Esposito V, Baldi A, Claudio PP, Fu Y, Caputi M, Pisano MM, Baldi F, Giordano A (1997) CDC2-related kinase PITALRE phosphorylates $\mathrm{pRb}$ exclusively on serine and is widely expressed in human tissues. J Cell Physiol 172:265-273. CrossRef Medline

Dincman TA, Beare JE, Ohri SS, Whittemore SR (2012) Isolation of cortical mouse oligodendrocyte precursor cells. J Neurosci Methods 209:219226. CrossRef Medline

Dugas JC, Cuellar TL, Scholze A, Ason B, Ibrahim A, Emery B, Zamanian JL, Foo LC, McManus MT, Barres BA (2010) Dicer1 and miR-219 Are required for normal oligodendrocyte differentiation and myelination. Neuron 65:597-611. CrossRef Medline

Durand B, Fero ML, Roberts JM, Raff MC (1998) p27Kip1 alters the response of cells to mitogen and is part of a cell-intrinsic timer that arrests the cell cycle and initiates differentiation. Curr Biol 8:431-440. CrossRef Medline

Emery B (2010) Transcriptional and post-transcriptional control of CNS myelination. Curr Opin Neurobiol 20:601-607. CrossRef Medline

Emery E, Aldana P, Bunge MB, Puckett W, Srinivasan A, Keane RW, Bethea J, Levi AD (1998) Apoptosis after traumatic human spinal cord injury. J Neurosurg 89:911-920. CrossRef Medline

ffrench-Constant C, Raff MC (1986) Proliferating bipotential glial progenitor cells in adult rat optic nerve. Nature 319:499-502. CrossRef Medline

Franklin RJ, Ffrench-Constant C (2008) Remyelination in the CNS: from biology to therapy. Nat Rev Neurosci 9:839-855. CrossRef Medline

Goldman SA, Osorio J (2014) So many progenitors, so little myelin. Nat Neurosci 17:483-485. CrossRef Medline

Graña X, De Luca A, Sang N, Fu Y, Claudio PP, Rosenblatt J, Morgan DO, Giordano A (1994) PITALRE, a nuclear CDC2-related protein kinase that phosphorylates the retinoblastoma protein in vitro. Proc Natl Acad Sci U S A 91:3834-3838. CrossRef Medline

Grimson A, Farh KK, Johnston WK, Garrett-Engele P, Lim LP, Bartel DP (2007) MicroRNA targeting specificity in mammals: determinants beyond seed pairing. Mol Cell 27:91-105. CrossRef Medline

Gudi V, Gingele S, Skripuletz T, Stangel M (2014) Glial response during cuprizone-induced de- and remyelination in the CNS: lessons learned. Front Cell Neurosci 8:73. CrossRef Medline 
He X, Yu Y, Awatramani R, Lu QR (2012) Unwrapping myelination by microRNAs. Neuroscientist 18:45-55. CrossRef Medline

Hibbits N, Yoshino J, Le TQ, Armstrong RC (2012) Astrogliosis during acute and chronic cuprizone demyelination and implications for remyelination. ASN Neuro 4:393-408. CrossRef Medline

Houbaviy HB, Murray MF, Sharp PA (2003) Embryonic stem cell-specific microRNAs. Dev Cell 5:351-358. CrossRef Medline

Jurevics H, Hostettler J, Muse ED, Sammond DW, Matsushima GK, Toews AD, Morell P (2001) Cerebroside synthesis as a measure of the rate of remyelination following cuprizone-induced demyelination in brain. J Neurochem 77:1067-1076. CrossRef Medline

Keirstead HS, Levine JM, Blakemore WF (1998) Response of the oligodendrocyte progenitor cell population (defined by NG2 labelling) to demyelination of the adult spinal cord. Glia 22:161-170. CrossRef Medline

Kim S, Kim JD, Chung AY, Kim HS, Kim YS, Kim MJ, Koun S, Lee YM, Rhee M, Park HC, Huh TL (2012) Antagonistic regulation of PAF1C and $\mathrm{p}-\mathrm{TEFb}$ is required for oligodendrocyte differentiation. J Neurosci 32: 8201-8207. CrossRef Medline

Kipp M, Clarner T, Dang J, Copray S, Beyer C (2009) The cuprizone animal model: new insights into an old story. Acta Neuropathol 118:723-736. CrossRef Medline

Kohoutek J (2009) P-TEFb- the final frontier. Cell Div 4:19. CrossRef Medline

Lau P, Verrier JD, Nielsen JA, Johnson KR, Notterpek L, Hudson LD (2008) Identification of dynamically regulated microRNA and mRNA networks in developing oligodendrocytes. J Neurosci 28:11720-11730. CrossRef Medline

Lewis BP, Shih IH, Jones-Rhoades MW, Bartel DP, Burge CB (2003) Prediction of mammalian microRNA targets. Cell 115:787-798. CrossRef Medline

Mallon BS, Shick HE, Kidd GJ, Macklin WB (2002) Proteolipid promoter activity distinguishes two populations of NG2-positive cells throughout neonatal cortical development. J Neurosci 22:876-885. Medline

Mason JL, Ye P, Suzuki K, D’Ercole AJ, Matsushima GK (2000) Insulin-like growth factor-1 inhibits mature oligodendrocyte apoptosis during primary demyelination. J Neurosci 20:5703-5708. Medline

Mason JL, Langaman C, Morell P, Suzuki K, Matsushima GK (2001) Episodic demyelination and subsequent remyelination within the murine central nervous system: changes in axonal calibre. Neuropathol Appl Neurobiol 27:50-58. CrossRef Medline

Matsushima GK, Morell P (2001) The neurotoxicant, cuprizone, as a model to study demyelination and remyelination in the central nervous system. Brain Pathol 11:107-116. CrossRef Medline

Miller DM, Rudick RA, Cutter G, Baier M, Fischer JS (2000) Clinical significance of the multiple sclerosis functional composite: relationship to patient-reported quality of life. Arch Neurol 57:1319-1324. CrossRef Medline

Morell P, Barrett CV, Mason JL, Toews AD, Hostettler JD, Knapp GW, Matsushima GK (1998) Gene expression in brain during cuprizone-induced demyelination and remyelination. Mol Cell Neurosci 12:220-227. CrossRef Medline

Peng J, Marshall NF, Price DH (1998) Identification of a cyclin subunit required for the function of Drosophila P-TEFb. J Biol Chem 273:1385513860. CrossRef Medline

Perlman SJ, Mar S (2012) Leukodystrophies. Adv Exp Med Biol 724:154171. CrossRef Medline
Redwine JM, Armstrong RC (1998) In vivo proliferation of oligodendrocyte progenitors expressing PDGFalphaR during early remyelination. J Neurobiol 37:413-428. CrossRef Medline

Reiner A, Yekutieli D, Benjamini Y (2003) Identifying differentially expressed genes using false discovery rate controlling procedures. Bioinformatics 19:368-375. CrossRef Medline

Simone C, Bagella L, Bellan C, Giordano A (2002) Physical interaction between $\mathrm{pRb}$ and cdk9/cyclinT2 complex. Oncogene 21:4158-4165. CrossRef Medline

Steelman AJ, Thompson JP, Li J (2012) Demyelination and remyelination in anatomically distinct regions of the corpus callosum following cuprizone intoxication. Neurosci Res 72:32-42. CrossRef Medline

Tang XM, Beesley JS, Grinspan JB, Seth P, Kamholz J, Cambi F (1999) Cell cycle arrest induced by ectopic expression of p27 is not sufficient to promote oligodendrocyte differentiation. J Cell Biochem 76:270-279. Medline

Vogel US, Reynolds R, Thompson RJ, Wilkin GP (1988) Expression of the $2^{\prime}, 3^{\prime}$-cyclic nucleotide $3^{\prime}$-phosphohydrolase gene and immunoreactive protein in oligodendrocytes as revealed by in situ hybridization and immunofluorescence. Glia 1:184-190. CrossRef Medline

Wang Q, Chow J, Hong J, Smith AF, Moreno C, Seaby P, Vrana P, Miri K, Tak J, Chung ED, Mastromonaco G, Caniggia I, Varmuza S (2011) Recent acquisition of imprinting at the rodent Sfmbt2 locus correlates with insertion of a large block of miRNAs. BMC Genomics 12:204. CrossRef Medline

Watson JV, Chambers SH, Smith PJ (1987) A pragmatic approach to the analysis of DNA histograms with a definable G1 peak. Cytometry 8:1-8. CrossRef Medline

Waxman SG (1989) Demyelination in spinal cord injury. J Neurol Sci 91:1-14. CrossRef Medline

Wegner M (2008) A matter of identity: transcriptional control in oligodendrocytes. J Mol Neurosci 35:3-12. CrossRef Medline

Wolswijk G, Noble M (1989) Identification of an adult-specific glial progenitor cell. Development 105:387-400. Medline

Wu CY, Lu J, Cao Q, Guo CH, Gao Q, Ling EA (2006) Expression of 2',3' cyclic nucleotide $3^{\prime}$-phosphodiesterase in the amoeboid microglial cells in the developing rat brain. Neuroscience 142:333-341. CrossRef Medline

Yang L, Kan EM, Lu J, Wu C, Ling EA (2014) Expression of 2',3'-cyclic nucleotide $3^{\prime}$-phosphodiesterase (CNPase) and its roles in activated microglia in vivo and in vitro. J Neuroinflammation 11:148. CrossRef Medline

Yang Z, He N, Zhou Q (2008) Brd4 recruits P-TEFb to chromosomes at late mitosis to promote G1 gene expression and cell cycle progression. Mol Cell Biol 28:967-976. CrossRef Medline

Ye P, Bagnell R, D’Ercole AJ (2003) Mouse NG2 + oligodendrocyte precursors express mRNA for proteolipid protein but not its DM-20 variant: a study of laser microdissection-captured NG2 + cells. J Neurosci 23:44014405. Medline

Yuan X, Chittajallu R, Belachew S, Anderson S, McBain CJ, Gallo V (2002) Expression of the green fluorescent protein in the oligodendrocyte lineage: a transgenic mouse for developmental and physiological studies. J Neurosci Res 70:529-545. CrossRef Medline

Zhao X, He X, Han X, Yu Y, Ye F, Chen Y, Hoang T, Xu X, Mi QS, Xin M, Wang F, Appel B, Lu QR (2010) MicroRNA-mediated control of oligodendrocyte differentiation. Neuron 65:612-626. CrossRef Medline 